SFB

Estimation of correlation for 823 continuous semimartingales

Mathias Vetter

Nr. 30/2010

OS

$\mathcal{O}$

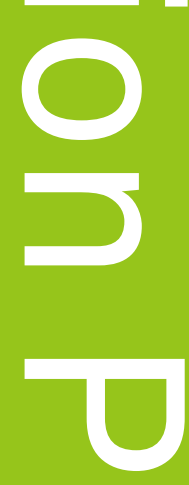

(1)

(D)

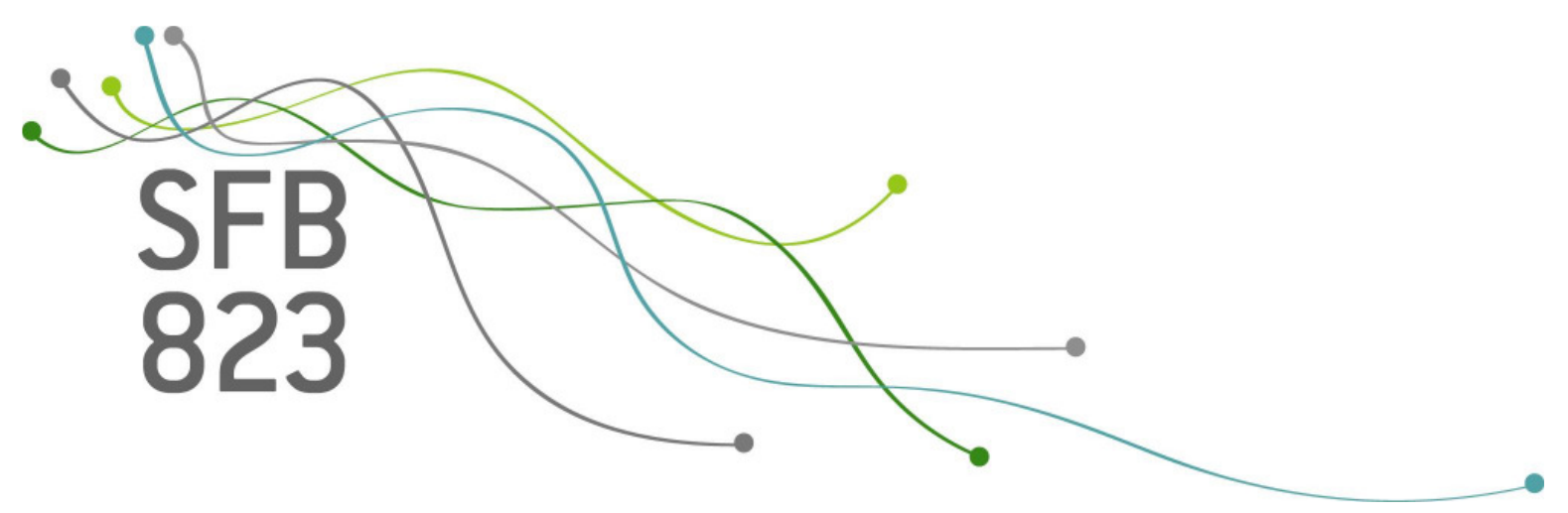





\title{
Estimation of correlation for continuous semimartingales
}

\author{
Mathias Vetter*
}

July 22, 2010

\begin{abstract}
In this paper we are concerned with inference on the correlation parameter $\rho$ of two Brownian motions, when only high-frequency observations from two one-dimensional continuous Itô semimartingales, driven by these particular Brownian motions, are available. Estimators for $\rho$ are constructed in two situations: Either when both components are observed (at the same time), or when only one component is observed and the other one represents its volatility process and thus has to be estimated from the data as well. In the first case it is shown that our estimator has the same asymptotic behaviour as the standard one for i.i.d. observations, whereas a feasible estimator can still be defined in the second framework, but with a slower rate of convergence.
\end{abstract}

Keywords: correlation, central limit theorem, high frequency observations, semimartingale.

AMS 2000 subject classifications: primary, 60F05, 62M07; secondary, 60G44.

${ }^{*}$ Ruhr-Universität Bochum, Fakultät für Mathematik, 44780 Bochum, Germany. Tel: +49-234-3223283. Fax: +49-234-3214559. Email: mathias.vetter@rub.de. The author is thankful for financial support through the collaborative research center "Statistical modeling of nonlinear dynamic processes" (SFB 823) of the German Research Foundation (DFG). 


\section{Introduction}

Recent years have seen a considerable progress in the statistics of processes observed at high frequency. For financial data, it is standard nowadays to assume that the underlying process, or a $\log$ transformed version, is given by a, possibly multidimensional, semimartingale $X$, and so natural issues both from a theoretical and an econometric perspective are the estimation of its quadratic (co)variation or of parts thereof, where particular interest has been turned towards inference on the integrated (co)volatility of the process, which plays a key role in important problems of mathematical finance (see [2], [4] or [9] among others). The proposed methods typically work for arbitrary Itô semimartingales, and they are model-free in the sense that neither a parametric setting for the diffusion coefficients is necessary nor that any properly rescaled limiting distribution depends on any of the coefficients involved.

On the other hand, when one turns towards statistical problems for a multidimensional semimartingale, and in particular when one inspects stochastic volatility models, there is one paramater that typically shows up in all of these models, namely the correlation coefficient between the driving Brownian motions. Since it is well-known that one is not able to draw inference about all coefficients of the underlying semimartingale at high frequency (most prominent is the drift part, which cannot be estimated apart from some special cases), it becomes a natural question, whether inference on this correlation parameter is in principle possible or not. Also, when one is concerned with applying such models in practice, both model calibration and model validation become important, and in this context estimation of correlation is obviously a standard issue as well. Hence, the aim of this paper is to provide simple (but feasible) estimators for the correlation structure of the underlying multidimensional Brownian motion, and for simplicity we restrict ourselves to the two-dimensional setting only, since its extension to similar frameworks in arbitrary dimensions is straight-forward.

Thus let us start with the following setting: Suppose, we are given a two-dimensional Itô semimartingale of the form

$$
\left(\begin{array}{c}
X_{t} \\
Y_{t}
\end{array}\right)=\int_{0}^{t}\left(\begin{array}{l}
a_{s} \\
b_{s}
\end{array}\right) d s+\int_{0}^{t}\left(\begin{array}{cc}
\sqrt{1-\rho^{2}} \sigma_{s} & \rho \sigma_{s} \\
0 & \tau_{s}
\end{array}\right) d\left(\begin{array}{l}
W_{s}^{1} \\
W_{s}^{2}
\end{array}\right)
$$

where $\left(W^{1}, W^{2}\right)^{T}$ is a two-dimensional standard Brownian motion, $a, b, \sigma$ and $\tau$ are appropriate stochastic processes, and $-1 \leq \rho \leq 1$ plays the role of a correlation coefficient between the Brownian motions that drive the one-dimensional processes $X$ and $Y$. This is visible from the alternative representation

$$
X_{t}=X_{0}+\int_{0}^{t} a_{s} d s+\int_{0}^{t} \sigma_{s} d W_{s} \quad \text { and } \quad Y_{t}=Y_{0}+\int_{0}^{t} b_{s} d s+\int_{0}^{t} \tau_{s} d V_{s}
$$

where $W$ and $V$ are jointly Brownian with $\operatorname{Corr}(W, V)=\rho$. We are interested in finding an estimator for $\rho$ (and in deriving its asymptotic properties), and we assume first that we are able to observe $X$ and $Y$ at the synchronous times $0,1 / n, \ldots, 1$ both. Of course, there is one restriction to be made, since

$$
\int_{0}^{t} \sigma_{s} d W_{s} \text { and } \int_{0}^{t}\left(-\sigma_{s}\right) d W_{s}=\int_{0}^{t} \sigma_{s} d(-W)_{s}
$$


follow the same distribution and thus identifiability becomes an issue. This problem can be overcome by assuming that $\sigma$ and $\tau$ are both almost surely positive, which is standard in finance. Nevertheless, in a more general setting only inference on $|\rho|$ is possible.

It turns out that it is indeed possible to obtain consistent estimates for $\rho$ in this framework, and under some mild additional assumptions we are able to prove an associated central limit theorem as well. The main idea behind the concept proposed in this paper is that increments of $X$ in a neighbourhood of some time $i / n$ can typically be approximated by the product of $\sigma_{\frac{i}{n}}$ with the corresponding increment of $W$. For this reason it is promising to divide the entire interval $[0,1]$ into subintervals of length $k_{n} / n$ for some appropriately chosen sequence of integers $k_{n}$. On each of these subintervals one computes the standard empirical correlation coefficient for mean zero observations based on increments of $X$ and $Y$, which gives a local estimate for the correlation. The final estimator $\tilde{\rho}$ for $\rho$ will then be given as the average of all local empirical coefficients. Surprisingly, we obtain a similar asymptotic behaviour of $\tilde{\rho}$ as for the empirical correlation coefficient for i.i.d. bivariate samples, namely

$$
\sqrt{n}(\tilde{\rho}-\rho) \stackrel{w}{\longrightarrow} \mathcal{N}\left(0, v^{2}\right)
$$

with $v^{2}=\left(1-\rho^{2}\right)^{2}$, which is the key result of this work.

But as indicated before, the typical situation for continuous Itô semimartingales in which inference about the correlation coefficient between two Brownian motions becomes important is when one investigates stochastic volatility models. In this case, one assumes that $X$ is given by (1.1) as before, but that there is an additional source of randomness that drives the volatility process $\sigma^{2}$ (or $\sigma$ ). Thus a natural model is that $\sigma^{2}$ is an Itô semimartingale itself with the representation

$$
\sigma_{t}^{2}=\sigma_{0}^{2}+\int_{0}^{t} b_{s} d s+\int_{0}^{t} \tau_{s} d V_{s}
$$

say, and where $W$ and $V$ are jointly Brownian with $\operatorname{Corr}(W, V)=\rho$ as before. In standard models both $b$ and $\tau$ come from specific parametric families, and most popular among these is the Heston model in which the volatility process is mean reverting and $\left(X, \sigma^{2}\right)^{T}$ satisfies

$$
X_{t}=X_{0}+\int_{0}^{t}\left(\mu-\frac{\sigma_{s}^{2}}{2}\right) d s+\int_{0}^{t} \sigma_{s} d W_{s} \quad \text { and } \quad \sigma_{t}^{2}=\sigma_{0}^{2}+\kappa \int_{0}^{t}\left(\alpha-\sigma_{s}^{2}\right) d s+\xi \int_{0}^{t} \sigma_{s} d V_{s}
$$

for some suitable parameters $\mu, \kappa, \alpha$ and $\xi$ (besides [7] see e.g. [8], [14] or [15] for other stochastic volatility models having continuous paths). In this context, $\rho$ is used to reproduce the well-known leverage effect of a (typically negative) correlation between stock returns and volatility.

For reasons of model building it is obvious that one may be interested in estimating any of the parameters involved, which is why we propose a general method for estimating the correlation between $W$ and $V$ in such stochastic volatility models. Since $\sigma^{2}$ is typically not observable and one can hardly assess the distribution of $X$ (or its increments) apart from special cases, one has to proceed in a different way as before. In contrast to [1], who propose to estimate the volatility process from observed option prices (and stock prices, of course), we will use a local volatility estimator from the stock price data only and plug those values into the estimator $\tilde{\rho}$, as if $\sigma^{2}$ were completely observable. Using this procedure we are able to prove a central limit theorem as well, but for which 
the rate of convergence becomes extremely low. Nevertheless, we provide some empirical examples in the section on simulations, which demonstrate that the proposed method has a reasonable finite sample behaviour in special cases as well, particularly when the correlation is zero.

Let us mention finally that our models neither deal with jumps in the processes nor take microstructure issues into account, which may seem to be a serious restriction. Note however, that in the last decade several methods have been developed to make inference about volatility processes even in the presence of jumps and noise possible, and straight-forward modifications of the presented statistics will lead to similar results as in the simpler models (1.1) and (1.2), but possibly with (further) losses in the rate of convergence. For details we refer to [5], [12], [16], [3] or [10].

This paper is organised as follows: Section 2 deals with the estimation of $\rho$ in model (1.1), whereas we discuss the more pronounced case for model (1.2) in Section 3. A small simulation study is presented in Section 4. All proofs are gathered in an Appendix.

\section{Inference for two discretely observed processes}

In this paragraph we discuss the case, in which the two continuous Itô semimartingales can be observed simultaneously. Precisely, suppose that we are given two one-dimensional continuous processes $X$ and $Y$, which are defined on the same filtered probability space $\left(\Omega, \mathcal{F},\left(\mathcal{F}_{t}\right)_{t \geq 0}, \mathbb{P}\right)$. Furthermore, we assume that both semimartingales have a representation as in (1.1), thus they can be written as

$$
X_{t}=X_{0}+\int_{0}^{t} a_{s} d s+\int_{0}^{t} \sigma_{s} d W_{s} \quad \text { and } \quad Y_{t}=Y_{0}+\int_{0}^{t} b_{s} d s+\int_{0}^{t} \tau_{s} d V_{s},
$$

where the two-dimensional Brownian motion $(W, V)$ satisfies $\operatorname{Corr}(W, V)=\rho$ and where both drift processes $a$ and $b$ are left continuous with right limits (and thus locally bounded). Furthermore, suppose that $\sigma$ is a continuous Itô semimartingales itself of the form

$$
\sigma_{t}=\sigma_{0}+\int_{0}^{t} v_{s} d s+\int_{0}^{t} \vartheta_{s}^{1} d W_{s}+\int_{0}^{t} \vartheta_{s}^{2} d V_{s}+\int_{0}^{t} \vartheta_{s}^{3} d U_{s}
$$

where $U$ is independent of $W$ and $V$, and that the processes above are chosen in such a way that $\sigma$ is almost surely positive. Additional technical assumptions are that the drift process $v$ is locally bounded and that the volatility processes $\vartheta^{i}$ are continuous Itô semimartingales as in (2.4) as well. Similar conditions are assumed to hold for $\tau$. As noted before, the interpretation in this situation is that $X$ and $Y$ represent the (log) price processes for two assets and that a practitioner might be interested in an analysis of their dependence structure in terms of the driving Brownian motions.

Throughout this section we assume further that we are able to observe both processes on the regular grid $t=0,1 / n, \ldots, 1$. When one assumes that both $X$ and $Y$ denote the price of some stock, this condition does not seem to be too restrictive, even though price processes of different assets are typically not observed at the same time. Nevertheless, there are ways to cope with this problem in practice (see e.g. [6]). The case, where $Y$ is the volatility process itself (and thus not observable), will be discussed in the following section. 
Our aim in this section is to define an estimator for the correlation parameter $\rho$ and to prove an associated central limit theorem. In order to do so we introduce an auxiliary sequence of integers $k_{n}$ satisfying

$$
k_{n}=c n^{\gamma}+o\left(n^{\gamma}\right) \quad \text { with } \quad \frac{1}{4}<\gamma<\frac{1}{2} \quad \text { and } \quad c>0
$$

which provides the size of the windows on which the local estimation of $\rho$ takes place. Explicitly, setting $\Delta_{j}^{n} T=T_{\frac{j}{n}}-T_{\frac{j-1}{n}}$ for any adapted process $T$, we define

$$
Z_{i}^{n}=\frac{\frac{1}{k_{n}} \sum_{j=i k_{n}+1}^{(i+1) k_{n}} \sqrt{n} \Delta_{j}^{n} X \sqrt{n} \Delta_{j}^{n} Y}{\sqrt{\frac{1}{k_{n}} \sum_{j=i k_{n}+1}^{(i+1) k_{n}}\left(\sqrt{n} \Delta_{j}^{n} X\right)^{2} \frac{1}{k_{n}} \sum_{j=i k_{n}+1}^{(i+1) k_{n}}\left(\sqrt{n} \Delta_{j}^{n} Y\right)^{2}}}
$$

for $i=0, \ldots\left\lfloor\frac{n}{k_{n}}\right\rfloor-1$, which is the local version of the empirical correlation coefficient in case of a known mean zero. Setting

$$
\tilde{\rho}=\frac{k_{n}}{n} \sum_{i=0}^{\left\lfloor\frac{n}{k_{n}}\right\rfloor-1}\left(Z_{i}^{n}+\frac{Z_{i}^{n}\left(1-\left(Z_{i}^{n}\right)^{2}\right)}{2\left(k_{n}-3\right)}\right)
$$

we obtain the following theorem, which is the main result of this section:

Theorem 2.1 Under the assumptions stated above we have

$$
\sqrt{n}(\tilde{\rho}-\rho) \stackrel{w}{\longrightarrow} \mathcal{N}\left(0, v^{2}\right)
$$

with $v^{2}=\left(1-\rho^{2}\right)^{2}$ and where $\stackrel{w}{\longrightarrow}$ denotes convergence in law.

Remark 2.2 Theorem 2.1 looks familiar, since it is well known that the empirical correlation coefficient

$$
\bar{\rho}=\frac{\frac{1}{n} \sum_{i=1}^{n} X_{i} Y_{i}}{\sqrt{\frac{1}{n} \sum_{i=1}^{n} X_{i}^{2} \frac{1}{n} \sum_{i=1}^{n} Y_{i}^{2}}},
$$

where the $\left(X_{i}, Y_{i}\right)$ are i.i.d. bivariate normally distributed random variables with $E\left[X_{i}\right]=E\left[Y_{i}\right]=0$ and covariance matrix

$$
\Sigma=\left(\begin{array}{ll}
1 & \rho \\
\rho & 1
\end{array}\right)
$$

satisfies $\sqrt{n}(\bar{\rho}-\rho) \stackrel{w}{\longrightarrow} \mathcal{N}\left(0, v^{2}\right)$ as well. Nevertheless, the difference between this standard coefficient $\bar{\rho}$ and our estimator $\tilde{\rho}$ is substantial, mainly for two reasons:

First, we are forced to estimate $\rho$ locally, since otherwise we do not know how to get rid of the dependence on the volatilities $\sigma$ and $\tau$. So the intuition behind $Z_{i}^{n}$ is that we have both

$$
\sqrt{n} \Delta_{j}^{n} X=\sigma_{\frac{i k_{n}}{n}} \sqrt{n} \Delta_{j}^{n} W+O_{p}\left(\sqrt{\frac{k_{n}}{n}}\right) \quad \text { and } \quad \sqrt{n} \Delta_{j}^{n} Y=\tau_{\frac{i k_{n}}{n}} \sqrt{n} \Delta_{j}^{n} V+O_{p}\left(\sqrt{\frac{k_{n}}{n}}\right)
$$


for $j$ close to $i k_{n}$, thus $Z_{i}^{n}$ becomes a local estimator for the correlation between $W$ and $V$, as in this case the volatilities $\sigma_{\frac{i k_{n}}{n}}$ and $\tau_{\frac{i k_{n}}{n}}$ cancel out. Afterwards, we use an average over all time points $i k_{n} / n$ to obtain a global estimator for $\rho$.

Note further that the correlation coefficient from (2.7) is not unbiased for $\rho$. For this reason we have to introduce the extra term in (2.6), which wipes out the first order term of the bias. Using this transformation is crucial for $\tilde{\rho}$ to achieve the same rate of convergence (and the same asymptotic distribution) as in the case of i.i.d. observations.

To summarize, the main idea behind Theorem 2.1 is the following: Setting

$$
\begin{aligned}
U_{i}^{n} & =\frac{\frac{1}{k_{n}} \sigma_{\frac{i k_{n}}{n}} \tau_{\frac{i k_{n}}{n}} \sum_{j=i k_{n}+1}^{(i+1) k_{n}} \sqrt{n} \Delta_{j}^{n} W \sqrt{n} \Delta_{j}^{n} V}{\sqrt{\frac{1}{k_{n}} \sum_{j=i k_{n}+1}^{(i+1) k_{n}} \sigma_{\frac{i k_{n}}{n}}^{2}\left(\sqrt{n} \Delta_{j}^{n} W\right)^{2} \frac{1}{k_{n}} \tau_{\frac{i k_{n}}{n}}^{2} \sum_{j=i k_{n}+1}^{(i+1) k_{n}}\left(\sqrt{n} \Delta_{j}^{n} V\right)^{2}}} \\
& =\frac{\frac{1}{k_{n}} \sum_{j=i k_{n}+1}^{(i+1) k_{n}} \sqrt{n} \Delta_{j}^{n} W \sqrt{n} \Delta_{j}^{n} V}{\sqrt{\frac{1}{k_{n}} \sum_{j=i k_{n}+1}^{(i+1) k_{n}}\left(\sqrt{n} \Delta_{j}^{n} W\right)^{2} \frac{1}{k_{n}} \sum_{j=i k_{n}+1}^{(i+1) k_{n}}\left(\sqrt{n} \Delta_{j}^{n} V\right)^{2}}},
\end{aligned}
$$

it can be shown from our choice of $k_{n}$ that both

$$
\sqrt{n} \frac{k_{n}}{n} \sum_{i=0}^{\left\lfloor\frac{n}{k_{n}}\right\rfloor-1}\left(Z_{i}^{n}-U_{i}^{n}\right)=o_{p}(1)
$$

and

$$
\frac{1}{\sqrt{n}} \sum_{i=0}^{\left\lfloor\frac{n}{k_{n}}\right\rfloor-1}\left(\left(Z_{i}^{n}\right)^{3}-\left(U_{i}^{n}\right)^{3}\right)=o_{p}(1)
$$

hold. Thus

$$
\sqrt{n} \frac{k_{n}}{n} \sum_{i=0}^{\left\lfloor\frac{n}{k_{n}}\right\rfloor-1}\left\{Z_{i}^{n}+\frac{Z_{i}^{n}\left(1-\left(Z_{i}^{n}\right)^{2}\right)}{2\left(k_{n}-3\right)}-\left(U_{i}^{n}+\frac{U_{i}^{n}\left(1-\left(U_{i}^{n}\right)^{2}\right)}{2\left(k_{n}-3\right)}\right)\right\}=o_{p}(1)
$$

follows, and we are left to prove

$$
\sqrt{n}\left(\frac{k_{n}}{n} \sum_{i=0}^{\left\lfloor\frac{n}{k_{n}}\right\rfloor-1}\left(U_{i}^{n}+\frac{U_{i}^{n}\left(1-\left(U_{i}^{n}\right)^{2}\right)}{2\left(k_{n}-3\right)}\right)-\rho\right) \stackrel{w}{\longrightarrow} \mathcal{N}\left(0, v^{2}\right),
$$

which brings us back to the familiar case of (a family of) empirical correlation coefficients for i.i.d. random variables.

For $|\rho| \neq 1$, Fisher's $z$-transformation makes a feasible version of Theorem 2.1 available. Thus, one can construct an asymptotic test for a given correlation coefficient $\rho_{0}$ with $-1<\rho_{0}<1$ easily.

Corollary 2.3 With $h(t)=\operatorname{artanh}(t)=\frac{1}{2} \log \left(\frac{1+t}{1-t}\right)$ we obtain

$$
\sqrt{n}(h(\tilde{\rho})-h(\rho)) \stackrel{w}{\longrightarrow} \mathcal{N}(0,1)
$$

for $-1<\rho<1$. 
Remark 2.4 When the correlation coefficient $\rho$ equals 1 (or -1 ), the asymptotic behaviour of $\tilde{\rho}$ becomes fundamentally different from the one of the standard estimator $\bar{\rho}$ for i.i.d. normal variables with mean zero and unit variance from (2.7). In fact, the latter statistic is almost surely constant and equal to +1 (or -1 ). For the estimator in our setting however, we only have the convergence in probability

$$
\sqrt{n}(\tilde{\rho}-\rho) \stackrel{P}{\longrightarrow} 0,
$$

due to the fact that $\tilde{\rho}$ contains additional randomness coming from the volatility, which is negligible at rate $n^{-\frac{1}{2}}$, but nevertheless present. In terms of $U_{i}^{n}$ from (2.8): For any choice of $k_{n}$ we have

$$
\sqrt{n} \frac{k_{n}}{n} \sum_{i=0}^{\left\lfloor\frac{n}{k_{n}}\right\rfloor-1}\left(\left(U_{i}^{n}+\frac{U_{i}^{n}\left(1-\left(U_{i}^{n}\right)^{2}\right)}{2\left(k_{n}-3\right)}\right)-\rho\right)=0
$$

almost surely, whereas

$$
\sqrt{n} \frac{k_{n}}{n} \sum_{i=0}^{\left\lfloor\frac{n}{k_{n}}\right\rfloor-1}\left\{Z_{i}^{n}+\frac{Z_{i}^{n}\left(1-\left(Z_{i}^{n}\right)^{2}\right)}{2\left(k_{n}-3\right)}-\left(U_{i}^{n}+\frac{U_{i}^{n}\left(1-\left(U_{i}^{n}\right)^{2}\right)}{2\left(k_{n}-3\right)}\right)\right\}=o_{p}(1)
$$

only under certain restrictions on $k_{n}$ as stated above. This suggests a possibility to derive a central limit theorem even in this case by choosing $k_{n}$ in such a way that the difference between $Z_{i}^{n}$ and $U_{i}^{n}$ drives the asymptotics, but an explicit investigation of this idea is beyond the scope of this paper.

\section{Inference for stochastic volatility models}

Recall model (1.1) and assume again that $X$ can be observed on the grid $t=0,1 / n, \ldots, 1$. In the second case discussed in this paper, $Y$ is the process $\sigma^{2}$ (or $\sigma$, which does not make a big difference) itself, and we assume that it takes the form

$$
\sigma_{t}^{2}=\sigma_{0}^{2}+\int_{0}^{t} b_{s} d s+\int_{0}^{t} \tau_{s} d V_{s}
$$

where its volatility process $\tau$ is given as in (2.4).

Remark 3.1 Note that $\sigma$ (which is the volatility process of $X$ ) does not have to satisfy (2.4) in general, but a localised version does. In fact, when the continuous process $\sigma^{2}$ is almost surely positive (which reduces to Feller's condition $2 \kappa \alpha>\xi^{2}$ for the Heston model (1.3)), the stopping times $T_{k}=\inf \left\{t>0: \sigma_{t}^{2} \leq 1 / k\right\}$ converge to infinity almost surely. However, for each $k$ there exists a smooth mapping $\phi_{k}$ that coincides with $x \mapsto \sqrt{x}$ on $(1 / k, \infty)$, and thus $\sigma_{s} 1_{\left[0, T_{k}\right)}(s)=$ $\phi_{k}\left(\sigma_{s}^{2}\right) 1_{\left[0, T_{k}\right)}(s)$ is of the form (2.4) from Itô's formula. As we are only interested in the process $\sigma$ on $[0,1]$, this is sufficient for our purposes. 
For a proper calibration of such a stochastic volatility model it is important to know about the correlation between $W$ and $V$. In contrast to the previous setting the volatility process $\sigma^{2}$ cannot be observed directly, and as we need to know about the local behaviour of it at time $k / n$, say, we propose to use some local estimators for it. Among several estimators, the most natural one appears to be the following: For an auxiliary sequence $m_{n}$ of integers, we set

$$
\hat{\sigma}_{\frac{k}{n}}^{2}=\frac{n}{m_{n}} \sum_{j=1}^{m_{n}}\left(X_{\frac{j+k}{n}}-X_{\frac{j+k-1}{n}}\right)^{2}
$$

for any $k=0, \ldots, n-m_{n}$. This definition is again justified by the fact that the volatility process does not fluctuate too much on a small interval and so $\left(\Delta_{j}^{n} X\right)^{2}$ becomes roughly $\sigma_{\frac{k m_{n}}{n}}^{2}\left(\Delta_{j}^{n} W\right)^{2}$. Precisely, we obtain the following result:

Lemma 3.2 For any sequence $m_{n}$ we have

$$
\hat{\sigma}_{\frac{k}{n}}^{2}-\sigma_{\frac{k}{n}}^{2}=O_{p}\left(\sqrt{m_{n} / n}+\sqrt{1 / m_{n}}\right),
$$

uniformly in $i$. The optimal choice is given for $m_{n}=O(\sqrt{n})$.

Sometimes we are interested in estimating $\sigma_{\frac{k}{n}}^{2}$ using increments from the left. In this case, one would use $\hat{\bar{\sigma}}_{\frac{k}{n}}^{2}=\hat{\sigma}_{\frac{k-m_{n}}{n}}^{2}$, for which a result similar to Lemma 3.2 holds.

Remark 3.3 Heuristically, it makes sense that one has to choose a moderate size for $m_{n}$ to obtain the optimal order in (3.13), as we have two competing sources of error. On one hand, we use an approximation of $\sigma_{s}^{2}$ by $\sigma_{\frac{i m_{n}}{n}}^{2}$, whose error can be shown to be of order $\sqrt{m_{n} / n}$, so it becomes smaller the smaller $m_{n}$ is. On the other hand, after replacing $\sigma_{s}^{2}$ by $\sigma_{\frac{i m_{n}}{n}}^{2}$, the estimate $\hat{\sigma}_{\frac{i m_{n}}{n}}^{2}-\sigma_{\frac{i m_{n}}{n}}^{2}$ becomes an average of $m_{n}$ terms with mean zero each, so its error is roughly $\sqrt{1 / m_{n}}$, and it becomes smaller the larger $m_{n}$ is.

There are several ways to construct a version of $\tilde{\rho}$, which takes into account that the volatility has to be estimated at certain time points. All of these have in common that their main structure is the same as before: We need increments of the process $X$, we need estimated increments of the process $\sigma^{2}$, and we need local estimators for the correlation coefficient. Therefore one has to define some auxiliary sequences of integers again, and we propose to use an estimator for which three (in general different) ones will be used: First, the sequence $m_{n}$ is used to define the local estimators from (3.12) for the volatility process $\sigma^{2}$, and we will go with the optimal choice from Lemma 3.2, that is

$$
m_{n}=b n^{\frac{1}{2}}+o\left(n^{\frac{1}{2}}\right) \quad \text { with } \quad b>0 .
$$

A further difference to the previous section arises from the fact that we need a second auxiliary sequence $l_{n}$ which governs the length of the intervals, on which the increments of $X$ and $\hat{\sigma}^{2}$ are computed. As we have to estimate increments of $\sigma^{2}$ (which are naturally of order $\sqrt{l_{n} / n}$ ) by 
increments of $\hat{\sigma}^{2}$, we have to choose the sequence $l_{n}$ in such a way that the approximation error due to the estimation of $\sigma^{2}$ (which is of order $\sqrt{m_{n} / n}$ ) is not dominating. It turns out that one can indeed take $l_{n}$ and $m_{n}$ to be equal, if one is only interested in a consistent estimation of $\rho$, whereas we choose $l_{n}$ to be

$$
l_{n}=d n^{\frac{2}{3}}+o\left(n^{\frac{2}{3}}\right) \quad \text { with } \quad d>0
$$

in order to obtain a central limit theorem. Finally, we still call $k_{n}$ the number of increments from which each local correlation coefficient is calculated, but its order will be different from the one in (2.5). Precisely, we set

$$
k_{n}=\tilde{c}\left(\frac{n}{l_{n}}\right)^{\tilde{\gamma}}+o\left(\left(\frac{n}{l_{n}}\right)^{\tilde{\gamma}}\right) \quad \text { with } \quad \frac{1}{4}<\tilde{\gamma}<\frac{1}{2} \quad \text { and } \quad \tilde{c}>0,
$$

thus of a similar order as the original from (2.5), but with the new effective sample size $n / l_{n}$.

The reason for this choice of $k_{n}$ is that we focus in this paper on a specific estimator $\hat{\rho}$, for which we split the entire interval $[0,1]$ into disjoint subintervals of length $l_{n} / n$. The data from each of these subintervals are used either for the estimation of $\sigma^{2}$ or to define an increment of the process $X$. This ensures a type of "conditional independence", which will be used in the proof of the central limit theorem later. Precisely, the construction goes as follows: Starting from zero we define blocks of size $3 l_{n} / n$, and on each block we compute three quantities:

(i) $\hat{\bar{\sigma}}_{\frac{(3 t-2) l_{n}}{n}}^{2}$, which serves as an estimator for $\frac{\sigma^{(3 t-2) l_{n}}}{n}$,

(ii) $X_{\frac{(3 t-1) l_{n}}{n}}-X_{\frac{(3 t-2) l_{n}}{n}}$,

(iii) $\hat{\sigma}_{\frac{(3 t-1) l_{n}}{n}}^{2}$, which serves as an estimator for $\frac{\sigma_{\frac{(3 t-1) l_{n}}{n}}^{2}}{n}$.

Here we have $t=1, \ldots,\left\lfloor\frac{n}{3 l_{n}}\right\rfloor$. As noted before, these quantities are all defined on non-overlapping intervals.

Now we are able to construct an analogue of $Z_{i}^{n}$, in which by construction everything happens on a different time scale as before. Since we use an interval of length $3 l_{n} / n$ to compute $X_{\frac{(3 k-1) l_{n}}{n}}-$ $X_{\frac{(3 k-2) l_{n}}{n}}$ and $\hat{\sigma}_{\frac{(3 k-1) l_{n}}{n}}^{2}-\hat{\bar{\sigma}}_{\frac{(3 k-2) l_{n}}{n}}^{2}$ on, the effective number of intervals will drop from $n$ in the completely observable case to $n /\left(3 l_{n}\right)$. This explains our choice of $k_{n}$, and thus we define $\hat{Z}_{n}^{i}$ for $i=0, \ldots,\left\lfloor\frac{n}{3 k_{n} l_{n}}\right\rfloor-1$ as follows:

$$
\hat{Z}_{n}^{i}=\frac{\frac{1}{k_{n}} \sum_{j=1}^{k_{n}} \frac{n}{l_{n}}\left(X_{\frac{\left(3 i k_{n}+3 j-1\right) l_{n}}{n}}-X_{\frac{\left(3 i k_{n}+3 j-2\right) l_{n}}{n}}\right)\left(\hat{\sigma}_{\frac{\left(3 i k_{n}+3 j-1\right) l_{n}}{n}}^{2}-\hat{\bar{\sigma}}_{\frac{\left(3 i k_{n}+3 j-2\right) l_{n}}{n}}^{2}\right)}{\sqrt{\frac{n}{k_{n} l_{n}} \sum_{j=1}^{k_{n}}\left(X_{\frac{\left(3 i k_{n}+3 j-1\right) l_{n}}{n}}-X_{\frac{\left(3 i k_{n}+3 j-2\right) l_{n}}{n}}\right)^{2} \frac{n}{k_{n} l_{n}} \sum_{j=1}^{k_{n}}\left(\hat{\sigma}_{\frac{\left(3 i k_{n}+3 j-1\right) l_{n}}{n}}^{2}-\hat{\bar{\sigma}}_{\frac{\left(3 i k_{n}+3 j-2\right) l_{n}}{n}}^{2}\right)^{2}}} .
$$

As before, we obtain an estimator for $\rho$ by setting

$$
\hat{\rho}=\frac{3 k_{n} l_{n}}{n} \sum_{i=0}^{\left\lfloor\frac{n}{3 k_{n} l_{n}}\right\rfloor-1}\left(\hat{Z}_{i}^{n}+\frac{\hat{Z}_{i}^{n}\left(1-\left(\hat{Z}_{i}^{n}\right)^{2}\right)}{2\left(k_{n}-3\right)}\right) .
$$

We come to the result on the asymptotic behaviour of $\hat{\rho}$. 
Theorem 3.4 Suppose that the assumptions stated above hold and that $l_{n}$ coincides with $m_{n}$ from (3.14). Then we have

$$
\hat{\rho} \stackrel{P}{\longrightarrow} \rho
$$

where $\stackrel{P}{\longrightarrow}$ denotes convergence in probability. Furthermore, if $\rho=0$ and $l_{n}$ is defined as in (3.15), the central limit theorem

$$
\sqrt{\frac{n}{3 l_{n}}} \hat{\rho} \stackrel{w}{\longrightarrow} \mathcal{N}(0,1)
$$

holds.

Remark 3.5 It is obvious that the variance in the central limit theorem above coincides with $v^{2}$ from Theorem 2.1 for $\rho=0$. Furthermore, one can prove that the relation

$$
\sqrt{\frac{n}{3 l_{n}}}(\hat{\rho}-\rho)=O_{p}(1)
$$

holds, even if the condition $\rho=0$ is not satisfied. In this case it should also be possible to derive a central limit theorem, but typically a non-vanishing bias will show up.

Remark 3.6 We have already seen that the rate of convergence in the central limit theorem has dropped from $n^{-\frac{1}{2}}$ in the completely observable case to $\left(l_{n} / n\right)^{-\frac{1}{2}}$ in this context, as it is governed by the number of increments from which the estimator for $\rho$ is constructed. However, it is not only the rate of convergence that becomes poor in this general framework. For finite samples the bigger problem appears to be the fact that the number of summands within $\hat{\rho}$ is of order $n /\left(k_{n} l_{n}\right)$, thus being of magnitude smaller than $n^{1 / 4}$, which means that it is at most about 10 for any reasonable choice of $n$. Nevertheless, we will see in the simulation study that the approximation from the central limit theorem still works quite well for finite samples.

\section{Simulation study}

In this section we provide a small simulation study assessing the finite sample properties of the estimators $\tilde{\rho}$ and $\hat{\rho}$. In order to have comparable results, we use the Heston model from (1.3) with the parameters $\mu=0.05 / 252, \kappa=5 / 252, \alpha=0.04 / 252$ and $\xi=0.5 / 252$ in both situations. All results are based on 10000 simulation runs.

We start with the first case, which means that we are in the situation that both the price process $X$ and the volatility process $\sigma^{2}$ can be observed at times $0,1 / \mathrm{n}, \ldots, 1$ (which is unrealistic in practice, of course). For the simulation study we define the sample sizes $n$ to be cubic, thus $k_{n}$ can and will simply be taken as $k_{n}=n^{1 / 3}$, which is in line with (2.5). For the sake of brevity we discuss the properties of the $z$-transformed statistic from Corollary 2.3 only, as the results for

$$
\frac{\sqrt{n}(\tilde{\rho}-\rho)}{1-\tilde{\rho}^{2}} \stackrel{w}{\longrightarrow} \mathcal{N}(0,1)
$$


from Theorem 2.1 (for $-1<\rho<1$ ) are similar.

\section{[INSERT TABLE 1 ABOUT HERE]}

We see from Table 1 that the approximation of the lower quantiles is satisfactory, even for moderate sample sizes. Note however that they appear to be slightly underestimated for a smaller value of $\rho$, whereas they become larger for a growing $\rho$. These findings are supported by additional simulations not reported here and are probably due to the special form of the volatility process in the Heston model. The approximation of the upper quantiles shows a similar picture, we is why we do not provide them explictly. Table 2 indicates that the proposed test has a high power even for small sample sizes, and that it is able to detect alternatives which are reasonably close to the null hypothesis. The results for a larger $n$ are omitted, as each null hypothesis is rejected in almost any case.

\section{[INSERT TABLE 2 ABOUT HERE]}

In the second situation, in which the price process $X$ is observed but the volatility is (as usual) not, we have more parameters to pick. We start with the consistency result from Theorem 3.4, and as this claim relies not only on $m_{n}=O\left(n^{1 / 2}\right)$, but also on $k_{n} \rightarrow \infty$ and $\frac{n}{m_{n} k_{n}} \rightarrow \infty$, we choose $m_{n}$ in general smaller than $n^{1 / 2}$. Reasonable values for any of the latter two quantities can only be obtained for large sample sizes, and we depict the precise choices for both sequences in Table 3. The simulation results show that the approximation of $\rho$ works in general well. Note however that the standard deviation is quite large in all cases, since both the correlation parameter and its estimator lie within $[-1,1]$ and thus the latter cannot deviate too much by construction. This explains also, why the standard deviation becomes smaller for a bigger absolute value of $\rho$, as a large deviation to one side becomes unlikely.

\section{[INSERT TABLE 3 ABOUT HERE]}

Finally, we investigate the finite sample performance of the $z$-transformed version of (3.18), and for the same reasons as before we set neither $m_{n}$ close to $n^{1 / 2}$ nor $l_{n}$ close to $n^{2 / 3}$. Also, as we do not want to throw away data and we only need intervals containing $m_{n}$ observations for the estimation of $\sigma^{2}$ (instead of $l_{n}$ data points as suggested implicitly in the definition of $\hat{\rho}$ ), we change $\hat{\rho}$ in the straight-forward way, making the effective sample size $\frac{n}{l_{n}+2 m_{n}}$. The exact choice for any of these auxiliary quantities is given in Table 4 . We see that the lower quantiles tend to be overestimated (the same holds for the upper quantiles, which are again not reported), but that this effect becomes smaller for a larger sample size, in which case the procedure yields passable results.

\section{[INSERT TABLE 4 ABOUT HERE]}

\section{Appendix}

As usual, standard localisation procedures as in [9] allow us to assume that any locally bounded process is actually bounded. Furthermore, we may also assume that the processes $\sigma^{2}$ and $\tau^{2}$ are bounded away from zero, so in particular we have $\sigma^{2}>K$ and $\tau^{2}>K$ for some $K>0$, which is 
fixed throughout the rest of this paper. Universal constants are denoted by $C$ or $C_{r}$, if we want to emphasize their dependencies on an additional parameter $r$.

Proof of Theorem 2.1 Recall the definition of $U_{i}^{n}$ in (2.8). Following Remark 2.2 we proceed in three steps. First, we show that we may replace $Z_{i}^{n}$ by the simpler $U_{i}^{n}$, which is (2.9) and (2.10), and later on we conclude with (2.11), which is obviously the easier part of the proof.

We divide the proof of (2.9) into two parts, by setting $Z_{i}^{n}-U_{i}^{n}=A_{i}^{n}+B_{i}^{n}$ with

$$
A_{i}^{n}=\frac{\frac{n}{k_{n}} \sum_{j=i k_{n}+1}^{(i+1) k_{n}}\left(\Delta_{j}^{n} X \Delta_{j}^{n} Y-\sigma_{\frac{i k_{n}}{n}} \tau_{\frac{i k_{n}}{n}} \Delta_{j}^{n} W \Delta_{j}^{n} V\right)}{\sqrt{\frac{n}{k_{n}} \sum_{j=i k_{n}+1}^{(i+1) k_{n}}\left(\Delta_{j}^{n} X\right)^{2} \frac{n}{k_{n}} \sum_{j=i k_{n}+1}^{(i+1) k_{n}}\left(\Delta_{j}^{n} Y\right)^{2}}}
$$

and

$$
B_{i}^{n}=\frac{\frac{n}{k_{n}} \sigma_{\frac{i k_{n}}{n}} \tau_{\frac{i k_{n}}{n}} \sum_{j=i k_{n}+1}^{(i+1) k_{n}} \Delta_{j}^{n} W \Delta_{j}^{n} V}{\sqrt{\frac{n}{k_{n}} \sum_{j=i k_{n}+1}^{(i+1) k_{n}}\left(\Delta_{j}^{n} X\right)^{2} \frac{n}{k_{n}} \sum_{j=i k_{n}+1}^{(i+1) k_{n}}\left(\Delta_{j}^{n} Y\right)^{2}}}-\frac{\frac{n}{k_{n}} \sum_{j=i k_{n}+1}^{(i+1) k_{n}} \Delta_{j}^{n} W \Delta_{j}^{n} V}{\sqrt{\frac{n}{k_{n}} \sum_{j=i k_{n}+1}^{(i+1) k_{n}}\left(\Delta_{j}^{n} W\right)^{2} \frac{n}{k_{n}} \sum_{j=i k_{n}+1}^{(i+1) k_{n}}\left(\Delta_{j}^{n} V\right)^{2}}} .
$$

Lemma 5.1 We have

$$
\sqrt{n} \frac{k_{n}}{n} \sum_{i=0}^{\left\lfloor\frac{n}{k_{n}}\right\rfloor-1} A_{i}^{n} \stackrel{P}{\longrightarrow} 0
$$

Proof of Lemma 5.1 Before we start with the proof, we have to introduce some additional notation. First, for any $1 \leq i \leq\left\lfloor\frac{n}{k_{n}}\right\rfloor$ we set

$$
\mu_{i}^{n}=\frac{n^{2}}{k_{n}^{2}} \sum_{j=i k_{n}+1}^{(i+1) k_{n}}\left(\Delta_{j}^{n} X\right)^{2} \sum_{j=i k_{n}+1}^{(i+1) k_{n}}\left(\Delta_{j}^{n} Y\right)^{2}, \quad \chi_{i}^{n}=\frac{n^{2}}{k_{n}^{2}} \sigma_{\frac{i k_{n}}{n}}^{2} \tau_{\frac{i k_{n}}{n}}^{2} \sum_{j=i k_{n}+1}^{(i+1) k_{n}}\left(\Delta_{j}^{n} W\right)^{2} \sum_{j=i k_{n}+1}^{(i+1) k_{n}}\left(\Delta_{j}^{n} V\right)^{2} .
$$

For later reasons, observe that the decomposition

$$
\Delta_{j}^{n} X \Delta_{j}^{n} Y-\sigma_{\frac{i k_{n}}{n}} \tau_{\frac{i k_{n}}{n}} \Delta_{j}^{n} W \Delta_{j}^{n} V=\left(\Delta_{j}^{n} X-\sigma_{\frac{i k_{n}}{n}} \Delta_{j}^{n} W\right) \Delta_{j}^{n} Y+\left(\Delta_{j}^{n} Y-\tau_{\frac{i k_{n}}{n}} \Delta_{j}^{n} V\right) \sigma_{\frac{i k_{n}}{n}} \Delta_{j}^{n} W
$$

for each $i k_{n}+1 \leq j \leq(i+1) k_{n}$ holds, and we need approximations for both quantities on the right hand side. (2.4) suggests that a reasonable estimate for the first one is given by

$$
M_{j}^{n}=\tau_{\frac{i k_{n}}{n}}\left(V_{\frac{j}{n}}-V_{\frac{j-1}{n}}\right) \int_{\frac{j-1}{n}}^{\frac{j}{n}}\left(\vartheta_{\frac{i k_{n}}{n}}^{1}\left(W_{s}-W_{\frac{i k_{n}}{n}}\right)+\vartheta_{\frac{i k_{n}}{n}}^{2}\left(V_{s}-V_{\frac{i k_{n}}{n}}\right)+\vartheta_{\frac{i k_{n}}{n}}^{3}\left(U_{s}-U_{\frac{i k_{n}}{n}}\right)\right) d W_{s},
$$

and a similar term $L_{j}^{n}$ can be defined for the second quantity as well. We write $A_{i}^{n}=P_{i}^{n}+Q_{i}^{n}$ with

$$
P_{i}^{n}=\frac{n}{k_{n}} \sum_{j=i k_{n}+1}^{(i+1) k_{n}}\left\{\left(\Delta_{j}^{n} X \Delta_{j}^{n} Y-\sigma_{\frac{i k_{n}}{n}} \tau_{\frac{i k_{n}}{n}} \Delta_{j}^{n} W \Delta_{j}^{n} V\right)-\left(L_{j}^{n}+M_{j}^{n}\right)\right\}
$$


and $Q_{i}^{n}$ defined implicitly. The proof will now consist of four steps: Once we have shown

$$
\begin{aligned}
& P\left(\min _{i} \sqrt{\mu_{i}^{n}}<K-\delta\right) \rightarrow 0 \text { for any } 0<\delta<K, \\
& \frac{k_{n}}{\sqrt{n}} \sum_{i=0}^{\left\lfloor\frac{n}{k_{n}}\right\rfloor-1} E\left[\left|P_{i}^{n}\right|\right] \rightarrow 0, \\
& \frac{k_{n}}{\sqrt{n}} \sum_{i=0}^{\left\lfloor\frac{n}{k_{n}}\right\rfloor-1} \frac{Q_{i}^{n}}{\sqrt{\chi_{i}^{n}}} \stackrel{P}{\longrightarrow} 0 \text { and } \\
& \frac{k_{n}}{\sqrt{n}} \sum_{i=0}^{\left\lfloor\frac{n}{k_{n}}\right\rfloor-1}\left(\frac{Q_{i}^{n}}{\sqrt{\mu_{i}^{n}}}-\frac{Q_{i}^{n}}{\sqrt{\chi_{i}^{n}}}\right) \stackrel{P}{\longrightarrow} 0,
\end{aligned}
$$

Lemma 5.1 follows from the following simple argument: For any $\varepsilon>0$ and some arbitrary but fixed $0<\delta<K$ we have

$$
\begin{aligned}
& P\left(\frac{k_{n}}{\sqrt{n}}\left|\sum_{i=0}^{\left\lfloor\frac{n}{k_{n}}\right\rfloor-1} A_{i}^{n}\right|>\varepsilon\right) \leq P\left(\frac{k_{n}}{\sqrt{n}}\left|\sum_{i=0}^{\left\lfloor\frac{n}{k_{n}}\right\rfloor-1} \frac{P_{i}^{n}}{\sqrt{\mu_{i}^{n}}}\right|>\frac{\varepsilon}{2}\right)+P\left(\frac{k_{n}}{\sqrt{n}}\left|\sum_{i=0}^{\left\lfloor\frac{n}{k_{n}}\right\rfloor-1} \frac{Q_{i}^{n}}{\sqrt{\mu_{i}^{n}}}\right|>\frac{\varepsilon}{2}\right) \\
\leq & P\left(\frac{k_{n}}{\sqrt{n}} \sum_{i=0}^{\left\lfloor\frac{n}{k_{n}}\right\rfloor-1} \frac{\left|P_{i}^{n}\right|}{\sqrt{\mu_{i}^{n}}}>\frac{\varepsilon}{2}, \min _{i} \sqrt{\mu_{i}^{n}} \geq K-\delta\right)+P\left(\min _{i} \sqrt{\mu_{i}^{n}}<K-\delta\right) \\
+ & P\left(\frac{k_{n}}{\sqrt{n}}\left|\sum_{i=0}^{\left\lfloor\frac{n}{k_{n}}\right\rfloor-1} \frac{Q_{i}^{n}}{\sqrt{\mu_{i}^{n}}}\right|>\frac{\varepsilon}{2}\right) .
\end{aligned}
$$

Now, the second term in the last display above converges to zero from (5.22), whereas the third one is small following (5.24) and (5.25), so we are left to focus on the first one. Note however that

$$
\begin{aligned}
& P\left(\frac{k_{n}}{\sqrt{n}} \sum_{i=0}^{\left\lfloor\frac{n}{k_{n}}\right\rfloor-1} \frac{\left|P_{i}^{n}\right|}{\sqrt{\mu_{i}^{n}}}>\frac{\varepsilon}{2}, \min _{i} \sqrt{\mu_{i}^{n}} \geq K-\delta\right) \\
\leq & P\left(\frac{k_{n}}{\sqrt{n}} \sum_{i=0}^{\left\lfloor\frac{n}{k_{n}}\right\rfloor-1} \frac{\left|P_{i}^{n}\right|}{K-\delta}>\frac{\varepsilon}{2}\right)=P\left(\frac{k_{n}}{\sqrt{n}} \sum_{i=0}^{\left\lfloor\frac{n}{k_{n}}\right\rfloor-1}\left|P_{i}^{n}\right|>\frac{\varepsilon(K-\delta)}{2}\right) \rightarrow 0
\end{aligned}
$$

from Markov's inequality and (5.23), and thus we are done.

Step 1: In the first step we prove (5.22). Recall the definition of $\mu_{i}^{n}$ from (5.19) and note that

$$
\begin{aligned}
& P\left(\min _{i} \sqrt{\mu_{i}^{n}}<K-\delta\right)=P\left(\min _{i} \mu_{i}^{n}<(K-\delta)^{2}\right) \\
\leq & P\left(\min _{i} \frac{n}{k_{n}} \sum_{j=i k_{n}+1}^{(i+1) k_{n}}\left(\Delta_{j}^{n} X\right)^{2}<K-\delta\right)+P\left(\min _{i} \frac{n}{k_{n}} \sum_{j=i k_{n}+1}^{(i+1) k_{n}}\left(\Delta_{j}^{n} Y\right)^{2}<K-\delta\right) .
\end{aligned}
$$

We will only prove

$$
P\left(\min _{i} \frac{n}{k_{n}} \sum_{j=i k_{n}+1}^{(i+1) k_{n}}\left(\Delta_{j}^{n} X\right)^{2}<K-\delta\right) \rightarrow 0
$$


as the second result can be obtained similarly. Observe that

$$
\begin{aligned}
& P\left(\min _{i} \frac{n}{k_{n}} \sum_{j=i k_{n}+1}^{(i+1) k_{n}}\left(\Delta_{j}^{n} X\right)^{2}<K-\delta\right) \leq \sum_{i=0}^{\left\lfloor\frac{n}{k_{n}}\right\rfloor-1} P\left(\frac{n}{k_{n}} \sum_{j=i k_{n}+1}^{(i+1) k_{n}}\left(\Delta_{j}^{n} X\right)^{2}<K-\delta\right) \\
\leq & \sum_{i=0}^{\left\lfloor\frac{n}{k_{n}}\right\rfloor-1} P\left(\frac{n}{k_{n}} \sum_{j=i k_{n}+1}^{(i+1) k_{n}}\left(\sigma_{\frac{i k_{n}}{n}} \Delta_{j}^{n} W\right)^{2}<K-\delta / 2\right) \\
+ & \sum_{i=0}^{\left\lfloor\frac{n}{k_{n}}\right\rfloor-1} P\left(\left|\frac{n}{k_{n}} \sum_{j=i k_{n}+1}^{(i+1) k_{n}}\left(\left(\Delta_{j}^{n} X\right)^{2}-\left(\sigma_{\frac{i k_{n}}{n}} \Delta_{j}^{n} W\right)^{2}\right)\right|>\delta / 2\right),
\end{aligned}
$$

and using $K<\sigma^{2}<C$ we conclude from Markov's inequality

$$
\begin{aligned}
P\left(\frac{n}{k_{n}} \sum_{j=i k_{n}+1}^{(i+1) k_{n}}\left(\sigma_{\frac{i k_{n}}{n}} \Delta_{j}^{n} W\right)^{2}<K-\delta / 2\right) & \leq P\left(\left|\frac{1}{k_{n}} \sum_{j=i k_{n}+1}^{(i+1) k_{n}} \sigma_{\frac{i k_{n}}{n}}^{2}\left(\left(\sqrt{n} \Delta_{j}^{n} W\right)^{2}-1\right)\right|>\delta / 2\right) \\
& \leq C\left(\frac{2}{\delta}\right)^{r} k_{n}^{-r / 2} E\left[\left|\frac{1}{\sqrt{k_{n}}} \sum_{j=i k_{n}+1}^{(i+1) k_{n}}\left(\left(\sqrt{n} \Delta_{j}^{n} W\right)^{2}-1\right)\right|^{r}\right]
\end{aligned}
$$

for any $r>0$. From the classical central limit theorem we know that the expectation on the right hand side of the last display is bounded by a constant which only depends on $r$, and thus

$$
\sum_{i=0}^{\left\lfloor\frac{n}{k_{n}}\right\rfloor-1} P\left(\frac{n}{k_{n}} \sum_{j=i k_{n}+1}^{(i+1) k_{n}}\left(\sigma_{\frac{i k_{n}}{n}} \Delta_{j}^{n} W\right)^{2}<K-\delta / 2\right) \rightarrow 0
$$

follows, since $k_{n}=c n^{\gamma}$ and $r$ can be chosen arbitrarily large. It remains to show

$$
\sum_{i=0}^{\left\lfloor\frac{n}{k_{n}}\right\rfloor-1} P\left(\left|\frac{n}{k_{n}} \sum_{j=i k_{n}+1}^{(i+1) k_{n}}\left(\left(\Delta_{j}^{n} X\right)^{2}-\left(\sigma_{\frac{i k_{n}}{n}} \Delta_{j}^{n} W\right)^{2}\right)\right|>\delta / 2\right) \rightarrow 0 .
$$

For the proof of (5.28) we use Itô's formula:

$$
\begin{aligned}
\left(\Delta_{j}^{n} X\right)^{2}-\left(\sigma_{\frac{i k_{n}}{n}} \Delta_{j}^{n} W\right)^{2}= & 2 \int_{\frac{j-1}{n}}^{\frac{j}{n}}\left(X_{s}-X_{\frac{j-1}{n}}\right) a_{s} d s+\int_{\frac{j-1}{n}}^{\frac{j}{n}}\left(\sigma_{s}^{2}-\sigma_{\frac{i k_{n}}{n}}^{2}\right) d s \\
& +2 \int_{\frac{j-1}{n}}^{\frac{j}{n}}\left\{\left(X_{s}-X_{\frac{j-1}{n}}\right) \sigma_{s}-\left(W_{s}-W_{\frac{j-1}{n}}\right) \sigma_{\frac{i k_{n}}{n}}^{2}\right\} d W_{s} .
\end{aligned}
$$

Let $r$ be an integer. From Hölder's inequality, $E\left[\left|\Delta_{i}^{n} X\right|^{2 r}\right] \leq C n^{-r}$ and the boundedness of $a$ one can conclude

$$
E\left[\left|\frac{n}{k_{n}} \sum_{j=i k_{n}+1}^{(i+1) k_{n}} \int_{\frac{j-1}{n}}^{\frac{j}{n}}\left(X_{s}-X_{\frac{j-1}{n}}\right) a_{s} d s\right|^{2 r}\right] \leq C n^{-r} .
$$


Since $\sigma$ is a continuous Itô semimartingale by assumption, we know from Itô's formula that $\sigma^{2}$ is of the same form. Therefore we have

$$
E\left[\left|\sigma_{s}^{2}-\sigma_{\frac{i k_{n}}{n}}^{2}\right|^{2 r}\right] \leq C\left(\frac{k_{n}}{n}\right)^{-r} \quad \text { for } \quad \frac{i k_{n}}{n} \leq s \leq \frac{(i+1) k_{n}}{n},
$$

and the same argument as before gives

$$
E\left[\left|\frac{n}{k_{n}} \sum_{j=i k_{n}+1}^{(i+1) k_{n}} \int_{\frac{j-1}{n}}^{\frac{j}{n}}\left(\sigma_{s}^{2}-\sigma_{\frac{i k_{n}}{n}}^{2}\right) d s\right|^{2 r}\right] \leq C\left(\frac{k_{n}}{n}\right)^{r} .
$$

Finally, note that

$$
\begin{aligned}
& E\left[\left|\left(X_{s}-X_{\frac{j-1}{n}}\right) \sigma_{s}-\left(W_{s}-W_{\frac{j-1}{n}}\right) \sigma_{\frac{i k_{n}}{n}}^{2}\right|^{r}\right] \\
\leq & C_{r} E\left[\left|\sigma_{s} \int_{\frac{j-1}{n}}^{s} a_{u} d u\right|^{r}+\left|\left(\sigma_{s}-\sigma_{\frac{i k_{n}}{n}}\right) \int_{\frac{j-1}{n}}^{s} \sigma_{u} d W_{u}\right|^{r}+\left|\sigma_{\frac{i k_{n}}{n}} \int_{\frac{j-1}{n}}^{s}\left(\sigma_{u}-\sigma_{\frac{i k_{n}}{n}}\right) d W_{u}\right|^{r}\right] .
\end{aligned}
$$

For $\frac{i k_{n}}{n} \leq s \leq \frac{(i+1) k_{n}}{n}$ various applications of Burkholder's and Hölder's inequality give

$$
\begin{aligned}
& E\left[\left|\sigma_{s} \int_{\frac{j-1}{n}}^{s} a_{u} d u\right|^{r}\right] \leq C_{r}\left(\frac{1}{n}\right)^{r}, \quad E\left[\left|\left(\sigma_{s}-\sigma_{\frac{i k_{n}}{n}}\right) \int_{\frac{j-1}{n}}^{s} \sigma_{u} d W_{u}\right|^{r}\right] \leq C_{r}\left(\frac{k_{n}}{n}\right)^{r / 2}\left(\frac{1}{n}\right)^{r / 2}, \\
& E\left[\left|\sigma_{\frac{i k_{n}}{n}} \int_{\frac{j-1}{n}}^{s}\left(\sigma_{u}-\sigma_{\frac{i k_{n}}{n}}\right) d W_{u}\right|^{r}\right] \leq C_{r}\left(\frac{k_{n}}{n}\right)^{r / 2}\left(\frac{1}{n}\right)^{r / 2},
\end{aligned}
$$

thus with

$$
g_{n}^{i}(s)=\frac{n}{k_{n}} \sum_{j=i k_{n}+1}^{(i+1) k_{n}} 1_{\left[\frac{j-1}{n}, \frac{j}{n}\right]}(s)\left\{\left(X_{s}-X_{\frac{j-1}{n}}\right) \sigma_{s}-\left(W_{s}-W_{\frac{j-1}{n}}\right) \sigma_{\frac{i k_{n}}{n}}^{2}\right\}
$$

we have

$$
\begin{aligned}
E\left[\left|g_{n}^{i}\right|^{r}(s)\right] & =\frac{n^{r}}{k_{n}^{r}} \sum_{j=i k_{n}+1}^{(i+1) k_{n}} 1_{\left[\frac{j-1}{n}, \frac{j}{n}\right]}(s) E\left[\left|\left(X_{s}-X_{\frac{j-1}{n}}\right) \sigma_{s}-\left(W_{s}-W_{\frac{j-1}{n}}\right) \sigma_{\frac{i k_{n}}{n}}^{2}\right|^{r}\right] \\
& \leq C_{r}\left(\frac{n}{k_{n}}\right)^{r}\left(\frac{k_{n}}{n}\right)^{r / 2}\left(\frac{1}{n}\right)^{r / 2} \sum_{j=i k_{n}+1}^{(i+1) k_{n}} 1_{\left[\frac{j-1}{n}, \frac{j}{n}\right]}(s)=C_{r} k_{n}^{-\frac{r}{2}} 1_{\left[\frac{i k_{n}}{n}, \frac{(i+1) k_{n}}{n}\right]}(s)
\end{aligned}
$$

for any $r>2$. Now

$$
\frac{n}{k_{n}} \sum_{j=i k_{n}+1}^{(i+1) k_{n}} \int_{\frac{j-1}{n}}^{\frac{j}{n}}\left\{\left(X_{s}-X_{\frac{j-1}{n}}\right) \sigma_{s}-\left(W_{s}-W_{\frac{j-1}{n}}\right) \sigma_{\frac{i k_{n}}{n}}^{2}\right\} d W_{s}=\int_{\frac{i k_{n}}{n}}^{\frac{(i+1) k_{n}}{n}} g_{n}^{i}(s) d W_{s},
$$

and from the same inequalities as before we conclude

$$
\begin{aligned}
E\left[\left|\int_{\frac{i k_{n}}{n}}^{\frac{(i+1) k_{n}}{n}} g_{n}^{i}(s) d W_{s}\right|^{r}\right] & \leq C_{r} E\left[\left.\left.\left|\int_{\frac{i k_{n}}{n}}^{\frac{(i+1) k_{n}}{n}}\right| g_{n}^{i}\right|^{2}(s) d s\right|^{r / 2}\right] \\
& \leq C_{r}\left(\frac{k_{n}}{n}\right)^{(r-2) / 2} E\left[\int_{\frac{i k_{n}}{n}}^{\frac{(i+1) k_{n}}{n}}\left|g_{n}^{i}\right|^{r}(s) d s\right] \leq C_{r} n^{-\frac{r}{2}}
\end{aligned}
$$


Putting together (5.30), (5.31) and (5.32), we obtain

$$
\sum_{i=0}^{\left\lfloor\frac{n}{k_{n}}\right\rfloor-1} P\left(\left|\frac{n}{k_{n}} \sum_{j=i k_{n}+1}^{(i+1) k_{n}}\left(\left(\Delta_{j}^{n} X\right)^{2}-\left(\sigma_{\frac{i k_{n}}{n}} \Delta_{j}^{n} W\right)^{2}\right)\right|>\delta / 2\right) \leq C\left(\frac{\delta}{2}\right)^{-2 r} \frac{n}{k_{n}}\left(\frac{k_{n}}{n}\right)^{r}
$$

from Markov's inequality, which converges to zero for $r$ large enough. This proves (5.28), so (5.22) as well.

Step 2: Now, we turn to the nominator of $A_{i}^{n}$. From (5.20) we know that for proving (5.23) it suffices to show

$$
\sqrt{n} \sum_{i=0}^{\left\lfloor\frac{n}{k_{n}}\right\rfloor-1} E\left[\left|\sum_{j=i k_{n}+1}^{(i+1) k_{n}}\left\{\left(\Delta_{j}^{n} X-\sigma_{\frac{i k_{n}}{n}} \Delta_{j}^{n} W\right) \Delta_{j}^{n} Y-M_{j}^{n}\right\}\right|\right] \rightarrow 0,
$$

as the related claim involving $L_{j}^{n}$ is easier to prove. Note that we have

$$
\left(\Delta_{j}^{n} X-\sigma_{\frac{i k_{n}}{n}} \Delta_{j}^{n} W\right) \Delta_{i}^{n} Y=\left(\int_{\frac{j-1}{n}}^{\frac{j}{n}} a_{s} d s+\int_{\frac{j-1}{n}}^{\frac{j}{n}}\left(\sigma_{s}-\sigma_{\frac{i k_{n}}{n}}\right) d W_{s}\right)\left(\int_{\frac{j-1}{n}}^{\frac{j}{n}} b_{s} d s+\int_{\frac{j-1}{n}}^{\frac{j}{n}} \tau_{s} d V_{s}\right)
$$

by definition of $Y$ and $X$. Proving

$$
\sqrt{n} \sum_{i=0}^{\left\lfloor\frac{n}{k_{n}}\right\rfloor-1} E\left[\left|\sum_{j=i k_{n}+1}^{(i+1) k_{n}}\left\{\left(\Delta_{j}^{n} X-\sigma_{\frac{i k_{n}}{n}} \Delta_{j}^{n} W\right) \int_{\frac{j-1}{n}}^{\frac{j}{n}} b_{s} d s\right\}\right|\right] \leq C \sqrt{\frac{k_{n}}{n}} \rightarrow 0
$$

is simple, and we have the decomposition

$$
\int_{\frac{j-1}{n}}^{\frac{j}{n}} a_{s} d s \int_{\frac{j-1}{n}}^{\frac{j}{n}} \tau_{s} d V_{s}=\sum_{l=1}^{3} \zeta(l)_{i, j}^{n}
$$

with

$$
\begin{aligned}
\zeta(1)_{i, j}^{n} & =\frac{1}{n} a_{\frac{i k_{n}}{n}} \tau_{\frac{i k_{n}}{n}} \Delta_{j}^{n} V, \quad \zeta(2)_{i, j}^{n}=\int_{\frac{j-1}{n}}^{\frac{j}{n}}\left(a_{s}-a_{\frac{i k_{n}}{n}}\right) d s \tau_{\frac{i k_{n}}{n}} \Delta_{j}^{n} V, \\
\zeta(3)_{i, j}^{n} & =\int_{\frac{j-1}{n}}^{\frac{j}{n}} a_{s} d s \int_{\frac{j-1}{n}}^{\frac{j}{n}}\left(\tau_{s}-\tau_{\frac{i k_{n}}{n}}\right) d V_{s} .
\end{aligned}
$$

We have $E\left[\zeta(1)_{i, j}^{n}\right]=0$ and $E\left[\left|\zeta(1)_{i, j}^{n}\right|^{2}\right] \leq C n^{-3}$ by successive conditioning. Thus,

$$
\sqrt{n} \sum_{i=0}^{\left\lfloor\frac{n}{k_{n}}\right\rfloor-1} E\left[\left|\sum_{j=i k_{n}+1}^{(i+1) k_{n}} \zeta(1)_{i, j}^{n}\right|\right] \leq \sqrt{n} \sum_{i=0}^{\left\lfloor\frac{n}{k_{n}}\right\rfloor-1} E\left[\left(\sum_{j=i k_{n}+1}^{(i+1) k_{n}} \zeta(1)_{i, j}^{n}\right)^{2}\right]^{\frac{1}{2}} \leq C \frac{1}{k_{n}^{1 / 2}} \rightarrow 0 .
$$


The second term satisfies

$$
\begin{aligned}
& \sqrt{n} \sum_{i=0}^{\left\lfloor\frac{n}{k_{n}}\right\rfloor-1} \sum_{j=i k_{n}+1}^{(i+1) k_{n}} E\left[\left|\zeta(2)_{i, j}^{n}\right|\right] \leq C \frac{1}{\sqrt{n}} \sum_{i=0}^{\left\lfloor\frac{n}{k_{n}}\right\rfloor-1} \sum_{j=i k_{n}+1}^{(i+1) k_{n}}\left(\int_{\frac{j-1}{n}}^{\frac{j}{n}} E\left[\left(a_{s}-a_{\frac{i k_{n}}{n}}\right)^{2}\right] d s\right)^{\frac{1}{2}} \\
& \leq C \sqrt{\frac{k_{n}}{n}} \sum_{i=0}^{\left\lfloor\frac{n}{k_{n}}\right\rfloor-1}\left(\sum_{j=i k_{n}+1}^{(i+1) k_{n}} \int_{\frac{j-1}{n}}^{\frac{j}{n}} E\left[\left(a_{s}-a_{\frac{i k_{n}}{n}}\right)^{2}\right] d s\right)^{\frac{1}{2}}
\end{aligned}
$$

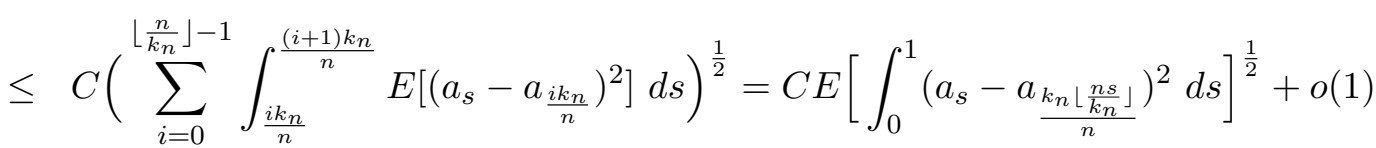

after various applications of Hölder's inequality. $a$ is left-continuous by assumption, so the integrand in the last display converges pointwise in $s$ to zero. Since $a$ is also bounded, an application of Lebesgue's theorem gives convergence to zero of the entire term as well. As before,

$$
E\left[\left|\zeta(3)_{i, j}^{n}\right|\right] \leq C \frac{\sqrt{k_{n}}}{n^{2}}, \quad \text { thus } \quad \sqrt{n} \sum_{i=0}^{\left\lfloor\frac{n}{k_{n}}\right\rfloor-1}\left|\sum_{j=i k_{n}+1}^{(i+1) k_{n}} \int_{\frac{j-1}{n}}^{\frac{j}{n}} a_{s} d s \int_{\frac{j-1}{n}}^{\frac{j}{n}} \tau_{s} d V_{s}\right| \stackrel{P}{\longrightarrow} 0,
$$

and it remains to show

$$
\sqrt{n} \sum_{i=0}^{\left\lfloor\frac{n}{k_{n}}\right\rfloor-1}\left|\sum_{j=i k_{n}+1}^{(i+1) k_{n}} \int_{\frac{j-1}{n}}^{\frac{j}{n}}\left(\sigma_{s}-\sigma_{\frac{i k_{n}}{n}}\right) d W_{s} \int_{\frac{j-1}{n}}^{\frac{j}{n}} \tau_{s} d V_{s}-M_{j}^{n}\right| \stackrel{P}{\longrightarrow} 0
$$

to obtain (5.33). Recall (2.4). Using Hölder's and Burkholder's inequality again, we obtain

$$
\sqrt{n} \sum_{i=0}^{\left\lfloor\frac{n}{k_{n}}\right\rfloor-1} \sum_{j=i k_{n}+1}^{(i+1) k_{n}} E\left[\left|\int_{\frac{j-1}{n}}^{\frac{j}{n}}\left(\int_{\frac{i k_{n}}{n}}^{s} v_{r} d r\right) d W_{s} \int_{\frac{j-1}{n}}^{\frac{j}{n}} \tau_{s} d V_{s}\right|\right] \leq C \frac{k_{n}}{\sqrt{n}},
$$

which converges to zero by assumption on $k_{n}$. At last,

$$
\int_{\frac{j-1}{n}}^{\frac{j}{n}}\left(\int_{\frac{i k_{n}}{n}}^{s} \vartheta_{r}^{1} d W_{r}+\int_{\frac{i k_{n}}{n}}^{s} \vartheta_{r}^{2} d V_{r}+\int_{\frac{i k_{n}}{n}}^{s} \vartheta_{r}^{3} d U_{r}\right) d W_{s} \int_{\frac{j-1}{n}}^{\frac{j}{n}} \tau_{s} d V_{s}-M_{j}^{n}
$$

can be decomposed into a sum of several quantities, each of which is of order $k_{n} / n^{2}$ from the same arguments as before. Since $k_{n} / \sqrt{n} \rightarrow 0$, this finishes the proof of (5.33).

Step 3: In this step we prove (5.24), and as before it suffices to show the result for the summand involving $M_{j}^{n}$ only. The main idea is to decompose $M_{j}^{n}$ into the sum of three terms, which can be written as a product of an $\mathcal{F}_{\frac{i k_{n}}{n}}$-measurable random variable and a functional of some multidimensional Brownian motion each. We only discuss the first one,

$$
\kappa_{j}^{n}=\frac{\vartheta_{\frac{i k_{n}}{n}}^{1}}{\sigma_{\frac{i k_{n}}{n}}} \frac{\int_{\frac{j-1}{n}}^{\frac{j}{n}}\left(W_{s}-W_{\frac{i k_{n}}{n}}\right) d W_{s}\left(V_{\frac{j}{n}}-V_{\frac{j-1}{n}}\right)}{\sqrt{\frac{1}{k_{n}} \sum_{j=i k_{n}+1}^{(i+1) k_{n}}\left(\sqrt{n} \Delta_{j}^{n} W\right)^{2} \frac{1}{k_{n}} \sum_{j=i k_{n}+1}^{(i+1) k_{n}}\left(\sqrt{n} \Delta_{j}^{n} V\right)^{2}}}
$$


for $i k_{n}+1 \leq j \leq(i+1) k_{n}$, thus we are left to prove

$$
\sqrt{n} \sum_{i=0}^{\left\lfloor\frac{n}{k_{n}}\right\rfloor-1} \sum_{j=i k_{n}+1}^{(i+1) k_{n}} \kappa_{j}^{n} \stackrel{P}{\longrightarrow} 0
$$

Now, recall that the inverse of a (central) chi-square distribution with $v$ degrees of freedom has finite moments for all integers $w<v / 2$. Precisely, a simple computation shows that if $N_{v} \sim \chi_{v}^{2}$, then

$$
E\left[N_{v}^{-w}\right]=\frac{\left(\frac{1}{2}\right)^{w}}{\left(\frac{v}{2}-1\right) \cdot \ldots \cdot\left(\frac{v}{2}-w\right)} \sim \frac{1}{v^{w}}
$$

for $v \rightarrow \infty$. Therefore we may from Hölder's and Burkholder's inequality plus (5.36) that

$$
E\left[\left|\kappa_{j}^{n}\right|^{2}\right] \leq k_{n}^{2} E\left[N_{k_{n}}^{-4}\right]^{1 / 2} E\left[\left(\int_{\frac{j-1}{n}}^{\frac{j}{n}}\left(W_{s}-W_{\frac{i k_{n}}{n}}\right) d W_{s} \Delta_{j}^{n} V\right)^{4}\right]^{1 / 2}<C \frac{k_{n}}{n^{3}}
$$

for $n$ large enough. Summarizing, $\kappa_{j}^{n}$ has finite first two moments for $n$ large enough, and it is also easy to see that "the Brownian part" of $\kappa_{j}^{n}$ (just as the one of the other two terms not reported here) is an odd functional in $(U, V, W)$. Since $(U, V, W) \stackrel{\mathcal{D}}{=}-(U, V, W)$, this implies $E\left[\kappa_{j}^{n} \mid \mathcal{F}_{\frac{i k_{n}}{n}}\right]=0$. Therefore, (5.37) and the assumptions on $\vartheta^{1}$ and $\sigma$ give

$E\left[\left(\sqrt{n} \sum_{i=0}^{\left\lfloor\frac{n}{k_{n}}\right\rfloor-1} \sum_{j=i k_{n}+1}^{(i+1) k_{n}} \kappa_{j}^{n}\right)^{2}\right] \leq C n \sum_{i=0}^{\left\lfloor\frac{n}{k_{n}}\right\rfloor-1} E\left[\left(\sum_{j=i k_{n}+1}^{(i+1) k_{n}} \kappa_{j}^{n}\right)^{2}\right]=C n \sum_{i=0}^{\left\lfloor\frac{n}{k_{n}}\right\rfloor-1} \sum_{j, l=i k_{n}+1}^{(i+1) k_{n}} E\left[\kappa_{j}^{n} \kappa_{l}^{n}\right] \rightarrow 0$

and this finishes the proof of (5.24).

Step 4: We are left to focus on (5.25), which finally implies Lemma 5.1. Our aim is to prove

$$
\frac{k_{n}}{\sqrt{n}} \sum_{i=0}^{\left\lfloor\frac{n}{k_{n}}\right\rfloor-1}\left(\frac{Q_{i}^{n}}{\sqrt{\chi_{i}^{n}}}-\frac{Q_{i}^{n}}{\sqrt{\mu_{i}^{n}}}\right)=\frac{k_{n}}{\sqrt{n}} \sum_{i=0}^{\left\lfloor\frac{n}{k_{n}}\right\rfloor-1} \frac{Q_{i}^{n}\left(\mu_{i}^{n}-\chi_{i}^{n}\right)}{\sqrt{\mu_{i}^{n}} \sqrt{\chi_{i}^{n}}\left(\sqrt{\mu_{i}^{n}}+\sqrt{\chi_{i}^{n}}\right)} \stackrel{P}{\longrightarrow} 0,
$$

and we can easily deduce

$$
P\left(\min _{i} \sqrt{\mu_{i}^{n}}<K-\delta\right) \rightarrow 0 \text { and } P\left(\min _{i} \sqrt{\chi_{i}^{n}}<K-\delta\right) \rightarrow 0
$$

for any $0<\delta<K$ from (5.22) and its proof. So with the same argument as in (5.26) it is sufficient to establish

$$
\frac{k_{n}}{\sqrt{n}} \sum_{i=0}^{\left\lfloor\frac{n}{k_{n}}\right\rfloor-1} E\left[\left|Q_{i}^{n}\left(\mu_{i}^{n}-\chi_{i}^{n}\right)\right|\right] \rightarrow 0
$$

We have

$$
\begin{aligned}
\mu_{i}^{n}-\chi_{i}^{n} & =\frac{n}{k_{n}} \sum_{j=i k_{n}+1}^{(i+1) k_{n}} \sigma_{\frac{i k_{n}}{n}}^{2}\left(\Delta_{j}^{n} W\right)^{2}\left(\frac{n}{k_{n}} \sum_{j=i k_{n}+1}^{(i+1) k_{n}}\left(\left(\Delta_{j}^{n} Y\right)^{2}-\tau_{\frac{i k_{n}}{n}}^{2}\left(\Delta_{j}^{n} V\right)^{2}\right)\right) \\
& +\frac{n}{k_{n}} \sum_{j=i k_{n}+1}^{(i+1) k_{n}}\left(\Delta_{j}^{n} Y\right)^{2}\left(\frac{n}{k_{n}} \sum_{j=i k_{n}+1}^{(i+1) k_{n}}\left(\left(\Delta_{j}^{n} X\right)^{2}-\sigma_{\frac{i k_{n}}{n}}^{2}\left(\Delta_{j}^{n} W\right)^{2}\right)\right)=: R_{i}^{n}+S_{i}^{n},
\end{aligned}
$$


and without loss of generality we will prove

$$
\frac{k_{n}}{\sqrt{n}} \sum_{i=0}^{\left\lfloor\frac{n}{k_{n}}\right\rfloor-1} E\left[\left|Q_{i}^{n} S_{i}^{n}\right|\right] \rightarrow 0
$$

only. Several applications of Hölder's and Burkholder's inequality give

$$
E\left[\left|Q_{i}^{n}\right|^{p}\right] \leq C_{p}\left(\frac{k_{n}}{n}\right)^{p / 2} \quad \text { and } \quad E\left[\left|\frac{n}{k_{n}} \sum_{j=i k_{n}+1}^{(i+1) k_{n}}\left(\Delta_{j}^{n} Y\right)^{2}\right|^{q}\right] \leq C_{q},
$$

for any two positive reals $p$ and $q$, and for an arbitrary positive $r$ we have

$$
E\left[\left|\frac{n}{k_{n}} \sum_{j=i k_{n}+1}^{(i+1) k_{n}}\left(\left(\Delta_{j}^{n} X\right)^{2}-\sigma_{\frac{i k_{n}}{n}}^{2}\left(\Delta_{j}^{n} W\right)^{2}\right)\right|^{r}\right] \leq C_{r}\left(\frac{k_{n}}{n}\right)^{\frac{r}{2}}
$$

from (5.30), (5.31) and (5.32) as well. Using the generalized version of Hölder's inequality we obtain

$$
\frac{k_{n}}{\sqrt{n}} \sum_{i=0}^{\left\lfloor\frac{n}{k_{n}}\right\rfloor-1} E\left[\left|Q_{i}^{n} S_{i}^{n}\right|\right] \leq C \sqrt{n} \frac{k_{n}}{n}=C \frac{k_{n}}{\sqrt{n}} \rightarrow 0
$$

and we are done.

Lemma 5.2 We have

$$
\sqrt{n} \frac{k_{n}}{n} \sum_{i=0}^{\left\lfloor\frac{n}{k_{n}}\right\rfloor-1} B_{i}^{n} \stackrel{P}{\longrightarrow} 0
$$

Proof of Lemma 5.2 This proof follows essentially the same strategy as the one of the previous lemma, and we will only give the main ideas. Recalling (5.19) and setting

$$
\gamma_{i}^{n}=\frac{n}{k_{n}} \sigma_{\frac{i k_{n}}{n}} \tau_{\frac{i k_{n}}{n}} \sum_{j=i k_{n}+1}^{(i+1) k_{n}} \Delta_{j}^{n} W \Delta_{j}^{n} V \quad \text { and } \quad \delta_{i}^{n}=\frac{n}{k_{n}} \sum_{j=i k_{n}+1}^{(i+1) k_{n}} \Delta_{j}^{n} X \Delta_{j}^{n} Y
$$

the assertion reduces to showing

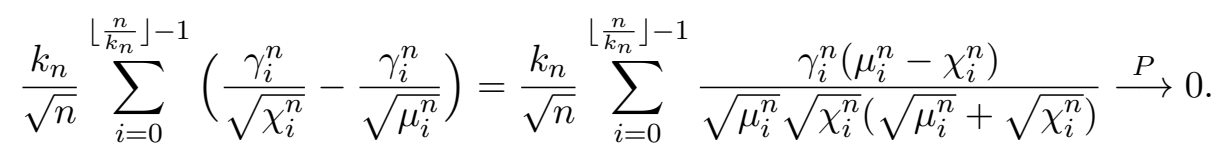

We have already discussed in (5.22) that the denominator is (in that particular sense) bounded from below in $n$, so the main focus will be on the nominator $\gamma_{i}^{n}\left(R_{i}^{n}+S_{i}^{n}\right)$ with the notation from (5.39).

As in the previous proof we need certain approximations for $\gamma_{i}^{n} R_{i}^{n}$ and $\gamma_{i}^{n} S_{i}^{n}$, which will be called 
$\theta_{i}^{n}$ and $\lambda_{i}^{n}$ in the following. For the sake of brevity we will only define and discuss $\lambda_{i}^{n}$ in detail, as the other quantity has a similar form and can be treated in the same way. Recalling (2.4) we set

$$
\lambda_{i}^{n}=\frac{n^{3}}{k_{n}^{3}} \sum_{j, k, l=i k_{n}+1}^{(i+1) k_{n}} \sigma_{\frac{i k_{n}}{n}} \tau_{\frac{i k_{n}}{n}}^{3} \Delta_{j}^{n} W \Delta_{j}^{n} V\left(\Delta_{k}^{n} V\right)^{2}\left(\alpha_{l}^{n}+\beta_{l}^{n}+\pi_{l}^{n}\right)
$$

with

$$
\alpha_{l}^{n}=2 a_{\frac{i k_{n}}{n}} \sigma_{\frac{i k_{n}}{n}} \int_{\frac{l-1}{n}}^{\frac{l}{n}}\left(W_{s}-W_{\frac{l-1}{n}}\right) d s, \quad \beta_{l}^{n}=\tau_{\frac{i k_{n}}{n}} \int_{\frac{l-1}{n}}^{\frac{l}{n}}\left(V_{s}-V_{\frac{i k_{n}}{n}}\right) d s,
$$

and

$$
\begin{aligned}
\pi_{l}^{n}=2 \int_{\frac{l-1}{n}}^{\frac{l}{n}}\left(a_{\frac{i k_{n}}{n}} \sigma_{\frac{i k_{n}}{n}} \int_{\frac{l-1}{n}}^{s} d u+\vartheta_{\frac{i k_{n}}{n}}^{1} \sigma_{\frac{i k_{n}}{n}}\left\{\left(W_{s}-W_{\frac{i k_{n}}{n}}\right) \int_{\frac{l-1}{n}}^{s} d W_{u}+\int_{\frac{l-1}{n}}^{s}\left(W_{u}-W_{\frac{i k_{n}}{n}}\right) d W_{u}\right\}\right. \\
+\vartheta_{\frac{i k_{n}}{n}}^{2} \sigma_{\frac{i k_{n}}{n}}\left\{\left(V_{s}-V_{\frac{i k_{n}}{n}}\right) \int_{\frac{l-1}{n}}^{s} d W_{u}+\int_{\frac{l-1}{n}}^{s}\left(V_{u}-V_{\frac{i k_{n}}{n}}\right) d W_{u}\right\} \\
\left.+\vartheta_{\frac{i k_{n}}{n}}^{3} \sigma_{\frac{i k_{n}}{n}}\left\{\left(U_{s}-U_{\frac{i k_{n}}{n}}\right) \int_{\frac{l-1}{n}}^{s} d W_{u}+\int_{\frac{l-1}{n}}^{s}\left(U_{u}-U_{\frac{i k_{n}}{n}}\right) d W_{u}\right\}\right) d W_{s}
\end{aligned}
$$

for $i k_{n}<l \leq(i+1) k_{n}$. Now, using the same arguments as in the proof of Lemma 5.1, we are finished once we have shown

$$
\begin{aligned}
& \frac{k_{n}}{\sqrt{n}} \sum_{i=0}^{\left\lfloor\frac{n}{k_{n}}\right\rfloor-1} E\left[\left|\gamma_{i}^{n}\left(\mu_{i}^{n}-\chi_{i}^{n}\right)-\left(\lambda_{i}^{n}+\theta_{i}^{n}\right)\right|\right] \rightarrow 0, \\
& \frac{k_{n}}{\sqrt{n}} \sum_{i=0}^{\left\lfloor\frac{n}{k_{n}}\right\rfloor-1} \frac{\lambda_{i}^{n}+\theta_{i}^{n}}{2\left(\chi_{i}^{n}\right)^{3 / 2}} \stackrel{P}{\longrightarrow} 0 \text { and } \\
& \frac{k_{n}}{\sqrt{n}} \sum_{i=0}^{\left\lfloor\frac{n}{k_{n}}\right\rfloor-1}\left(\frac{\lambda_{i}^{n}+\theta_{i}^{n}}{\sqrt{\mu_{i}^{n}} \sqrt{\chi_{i}^{n}}\left(\sqrt{\mu_{i}^{n}}+\sqrt{\chi_{i}^{n}}\right)}-\frac{\lambda_{i}^{n}+\theta_{i}^{n}}{2\left(\chi_{i}^{n}\right)^{3 / 2}}\right) \stackrel{P}{\longrightarrow} 0,
\end{aligned}
$$

where we have used (5.38).

As said before, instead of proving (5.44) we discuss

$$
\frac{k_{n}}{\sqrt{n}} \sum_{i=0}^{\left\lfloor\frac{n}{k_{n}}\right\rfloor-1} E\left[\left|\gamma_{i}^{n} S_{i}^{n}-\lambda_{i}^{n}\right|\right] \rightarrow 0
$$

only. We have

$$
\gamma_{i}^{n} S_{i}^{n}=\frac{n^{3}}{k_{n}^{3}} \sum_{j, k, l=i k_{n}+1}^{(i+1) k_{n}} \sigma_{\frac{i k_{n}}{n}} \tau_{\frac{i k_{n}}{n}} \Delta_{j}^{n} W \Delta_{j}^{n} V\left(\Delta_{k}^{n} Y\right)^{2}\left(\left(\Delta_{l}^{n} X\right)^{2}-\sigma_{\frac{i k_{n}}{n}}^{2}\left(\Delta_{l}^{n} W\right)^{2}\right),
$$


thus using $E\left[\left|\Delta_{j}^{n} W\right|^{r}\right] \leq C n^{-\frac{r}{2}},(5.41)$ twice (once in terms of $X$ and once in terms of $\sigma^{2}$ ) plus Hölder's inequality we obtain

$$
E\left[\left|\gamma_{i}^{n} S_{i}^{n}-\frac{n^{3}}{k_{n}^{3}} \sum_{j, k, l=i k_{n}+1}^{(i+1) k_{n}} \sigma_{\frac{i k_{n}}{n}} \tau_{\frac{i k_{n}}{n}}^{3} \Delta_{j}^{n} W \Delta_{j}^{n} V\left(\Delta_{k}^{n} V\right)^{2}\left(\left(\Delta_{l}^{n} X\right)^{2}-\sigma_{\frac{i k_{n}}{n}}^{2}\left(\Delta_{l}^{n} W\right)^{2}\right)\right|\right] \leq C \frac{k_{n}}{n} .
$$

With $k_{n} / \sqrt{n} \rightarrow 0$ and from the boundedness of $\sigma$ and $\tau$, (5.47) reduces to showing that

$$
\frac{n^{5 / 2}}{k_{n}^{2}} \sum_{i=0}^{\left\lfloor\frac{n}{k_{n}}\right\rfloor-1} E\left[\left|\sum_{j, k, l=i k_{n}+1}^{(i+1) k_{n}} \Delta_{j}^{n} W \Delta_{j}^{n} V\left(\Delta_{k}^{n} V\right)^{2}\left(\left(\Delta_{l}^{n} X\right)^{2}-\sigma_{\frac{i k_{n}}{n}}^{2}\left(\Delta_{l}^{n} W\right)^{2}-\left(\alpha_{l}^{n}+\beta_{l}^{n}+\pi_{l}^{n}\right)\right)\right|\right]
$$

converges to zero. To this end, we use the following estimates which are straight-forward consequences of Hölder's and Burkholder's inequality:

$$
\begin{aligned}
& E\left[\left|2 \int_{\frac{j-1}{n}}^{\frac{j}{n}}\left(X_{s}-X_{\frac{j-1}{n}}\right) a_{s} d s-\alpha_{j}^{n}\right|^{2}\right] \leq C\left(\frac{1}{n^{2}} E\left[\int_{\frac{l-1}{n}}^{\frac{l}{n}}\left(a_{s}-a_{\frac{i k_{n}}{n}}\right)^{2} d s\right]+\frac{k_{n}}{n^{4}}\right), \\
& E\left[\left|\int_{\frac{j-1}{n}}^{\frac{j}{n}}\left(\sigma_{s}^{2}-\sigma_{\frac{i k_{n}}{n}}^{2}\right) d s-\beta_{j}^{n}\right|^{2}\right] \leq C \frac{k_{n}^{2}}{n^{4}} \\
& E\left[\left|2 \int_{\frac{j-1}{n}}^{\frac{j}{n}}\left\{\left(X_{s}-X_{\frac{j-1}{n}}\right) \sigma_{s}-\left(W_{s}-W_{\frac{j-1}{n}}\right) \sigma_{\frac{i k_{n}}{n}}^{2}\right\} d W_{s}-\pi_{j}^{n}\right|^{2}\right] \\
& \leq C\left(\frac{1}{n^{2}} E\left[\int_{\frac{l-1}{n}}^{\frac{l}{n}}\left(a_{s}-a_{\frac{i k_{n}}{n}}\right)^{2} d s\right]+\frac{k_{n}^{2}}{n^{4}}\right) .
\end{aligned}
$$

Thus we may conclude from (5.29) that

$$
\begin{gathered}
E\left[\left|\sum_{j, k, l=i k_{n}+1}^{(i+1) k_{n}} \Delta_{j}^{n} W \Delta_{j}^{n} V\left(\Delta_{k}^{n} V\right)^{2}\left(\sigma_{\frac{i k_{n}}{n}}^{2}\left(\Delta_{l}^{n} W\right)^{2}-\left(\Delta_{l}^{n} X\right)^{2}-\left(\alpha_{l}^{n}+\beta_{l}^{n}+\pi_{l}^{n}\right)\right)\right|\right] \\
\leq C \frac{k_{n}^{3}}{n^{3}}\left(E\left[\int_{\frac{l-1}{n}}^{\frac{l}{n}}\left(a_{s}-a_{\frac{i k_{n}}{n}}\right)^{2} d s\right]^{1 / 2}+\frac{k_{n}}{n}\right),
\end{gathered}
$$

and the proof of (5.47) is complete, once we use again the fact that $a$ is left-continuous.

Showing (5.45) is similar to proving (5.24): We know from Hölder's inequality that $\frac{\lambda_{i}^{n}+\theta_{i}^{n}}{2\left(\chi_{i}^{n}\right)^{3 / 2}}$ has finite first moments for $n$ large enough. Now, have a look at $\frac{\lambda_{i}^{n}}{2\left(\chi_{i}^{n}\right)^{3 / 2}}$ for example: Similar to the definition of $\kappa_{i}^{n}$ in the proof of (5.24) $\mathcal{F}_{\frac{i k_{n}}{n}}$-measurable variables do not matter here as well. Also, the resulting variable has finite first moments, and using Hölder's inequality and (5.36) we obtain

$$
E\left[\left(\frac{\lambda_{i}^{n}+\theta_{i}^{n}}{2\left(\chi_{i}^{n}\right)^{3 / 2}}\right)^{r}\right] \leq C E\left[\left(\lambda_{i}^{n}+\theta_{i}^{n}\right)^{2 r}\right]^{1 / 2} \leq C\left(\frac{k_{n}}{n}\right)^{r / 2}
$$

for any integer $r$. As the variable is also odd in $(U, V, W)$ (each of the quantities $\alpha_{i}^{n}, \beta_{i}^{n}$ and $\pi_{i}^{n}$ is), its first moment is zero, and

$$
E\left[\left(\frac{k_{n}}{\sqrt{n}} \sum_{i=0}^{\left\lfloor\frac{n}{k_{n}}\right\rfloor-1} \frac{\lambda_{i}^{n}+\theta_{i}^{n}}{2\left(\chi_{i}^{n}\right)^{3 / 2}}\right)^{2}\right]=\frac{k_{n}^{2}}{n} \sum_{i=0}^{\left\lfloor\frac{n}{k_{n}}\right\rfloor-1} E\left[\left(\frac{\lambda_{i}^{n}+\theta_{i}^{n}}{2\left(\chi_{i}^{n}\right)^{3 / 2}}\right)^{2}\right] \leq C \frac{k_{n}^{2}}{n} \rightarrow 0 .
$$


Finally,

$$
\begin{aligned}
& \frac{\lambda_{i}^{n}+\theta_{i}^{n}}{\sqrt{\mu_{i}^{n}} \sqrt{\chi_{i}^{n}}\left(\sqrt{\mu_{i}^{n}}+\sqrt{\chi_{i}^{n}}\right)}-\frac{\lambda_{i}^{n}+\theta_{i}^{n}}{2\left(\chi_{i}^{n}\right)^{3 / 2}} \\
= & \frac{\left(\lambda_{i}^{n}+\theta_{i}^{n}\right)\left(\chi_{i}^{n}-\mu_{i}^{n}\right)}{2\left(\chi_{i}^{n}\right)^{3 / 2} \sqrt{\mu_{i}^{n}}\left(\sqrt{\mu_{i}^{n}}+\sqrt{\chi_{i}^{n}}\right)}+\frac{\left(\lambda_{i}^{n}+\theta_{i}^{n}\right) \sqrt{\chi_{i}^{n}}\left(\chi_{i}^{n}-\mu_{i}^{n}\right)}{2\left(\chi_{i}^{n}\right)^{3 / 2} \sqrt{\mu_{i}^{n}}\left(\sqrt{\mu_{i}^{n}}+\sqrt{\chi_{i}^{n}}\right)^{2}},
\end{aligned}
$$

and using (5.38) we are left to prove

$$
\frac{k_{n}}{\sqrt{n}} \sum_{i=0}^{\left\lfloor\frac{n}{k_{n}}\right\rfloor-1} E\left[\left|\left(\lambda_{i}^{n}+\theta_{i}^{n}\right)\left(\chi_{i}^{n}-\mu_{i}^{n}\right)\left(\sqrt{\chi_{i}^{n}}+1\right)\right|\right] \rightarrow 0
$$

as before. Using (5.49), (5.41) and $E\left[\left|\chi_{i}^{n}\right|^{r}\right] \leq C_{r}$ we obtain

$$
E\left[\left|\lambda_{i}^{n}+\theta_{i}^{n}\right|^{3}\right]^{1 / 3} \leq C\left(\frac{k_{n}}{n}\right)^{1 / 2}, \quad E\left[\left|\chi_{i}^{n}-\mu_{i}^{n}\right|^{3}\right]^{1 / 3} \leq C\left(\frac{k_{n}}{n}\right)^{1 / 2}, \quad E\left[\left|\sqrt{\chi_{i}^{n}}+1\right|^{3}\right]^{1 / 3} \leq C,
$$

and thus Hölder's inequality and $k_{n} / \sqrt{n} \rightarrow 0$ yield (5.51).

The proof of (2.10) and (2.11) is simpler. For the first result, note that

$$
\left(Z_{i}^{n}\right)^{3}-\left(U_{i}^{n}\right)^{3}=\left(Z_{i}^{n}\right)^{2}\left(Z_{i}^{n}-U_{i}^{n}\right)+Z_{i}^{n} U_{i}^{n}\left(Z_{i}^{n}-U_{i}^{n}\right)+\left(U_{i}^{n}\right)^{2}\left(Z_{i}^{n}-U_{i}^{n}\right),
$$

and without loss of generality we discuss the first term on the right hand side only. Recall (5.42). We have

$$
\left(Z_{i}^{n}\right)^{2}\left(Z_{i}^{n}-U_{i}^{n}\right)=\frac{\left(\delta_{i}^{n}\right)^{2}}{\mu_{i}^{n}}\left(\frac{\delta_{i}^{n}}{\sqrt{\mu_{i}^{n}}}-\frac{\gamma_{i}^{n}}{\sqrt{\chi_{i}^{n}}}\right)=\frac{\left(\delta_{i}^{n}\right)^{3}\left(\chi_{i}^{n}-\mu_{i}^{n}\right)}{\left(\mu_{i}^{n}\right)^{3 / 2} \sqrt{\chi_{i}^{n}}\left(\sqrt{\chi_{i}^{n}}+\sqrt{\mu_{i}^{n}}\right)}+\frac{\left(\delta_{i}^{n}\right)^{2}\left(\delta_{i}^{n}-\gamma_{i}^{n}\right)}{\mu_{i}^{n} \sqrt{\chi_{i}^{n}}} .
$$

Using (5.38) we may conclude as in the previous proof that (2.10) follows, once we have established

$$
\frac{1}{\sqrt{n}} \sum_{i=0}^{\left\lfloor\frac{n}{k_{n}}\right\rfloor-1} E\left[\left|\left(\delta_{i}^{n}\right)^{3}\left(\chi_{i}^{n}-\mu_{i}^{n}\right)\right|+\left|\left(\delta_{i}^{n}\right)^{2}\left(\delta_{i}^{n}-\gamma_{i}^{n}\right)\right|\right] \rightarrow 0
$$

The same arguments that lead to (5.41) give both

$$
E\left[\left|\chi_{i}^{n}-\mu_{i}^{n}\right|^{r}\right] \leq C_{r}\left(\frac{k_{n}}{n}\right)^{r / 2} \text { and } E\left[\left|\delta_{i}^{n}-\gamma_{i}^{n}\right|^{r}\right] \leq C_{r}\left(\frac{k_{n}}{n}\right)^{r / 2},
$$

and it is easy to obtain $E\left[\left|\delta_{i}^{n}\right|^{r}\right] \leq C_{r}$ and $E\left[\left|\mu_{i}^{n}\right|^{r}\right] \leq C_{r}$. Applying $k_{n} \rightarrow \infty$ completes the proof of (5.53).

It remains to prove (2.11). Since the $U_{i}^{n}$ form a triangular array of rowwise independent and identically distributed random variables, this claim follows from

$$
\sqrt{n}\left(E\left[U_{1}^{n}+\frac{U_{1}^{n}\left(1-\left(U_{i}^{n}\right)^{2}\right)}{2\left(k_{n}-3\right)}\right]-\rho\right) \rightarrow 0
$$


and

$$
k_{n} \operatorname{Var}\left(U_{1}^{n}+\frac{U_{1}^{n}\left(1-\left(U_{i}^{n}\right)^{2}\right)}{2\left(k_{n}-3\right)}\right) \rightarrow v^{2} .
$$

From equation (2.6) in [13] we know that

$$
E\left[U_{1}^{n}+\frac{U_{1}^{n}\left(1-\left(U_{i}^{n}\right)^{2}\right)}{2\left(k_{n}-3\right)}\right]-\rho=O\left(k_{n}^{-2}\right),
$$

and (5.54) follows, since $n^{1 / 2} k_{n}^{-2} \rightarrow 0$ by assumption. (5.55) is a standard consequence of the Cramér-Wold theorem. Setting

$$
F_{i}^{n}=\frac{n}{k_{n}} \sum_{j=i k_{n}+1}^{(i+1) k_{n}} \Delta_{j}^{n} W \Delta_{j}^{n} V, \quad G_{i}^{n}=\frac{n}{k_{n}} \sum_{j=i k_{n}+1}^{(i+1) k_{n}}\left(\Delta_{j}^{n} W\right)^{2}, \quad H_{i}^{n}=\frac{n}{k_{n}} \sum_{j=i k_{n}+1}^{(i+1) k_{n}}\left(\Delta_{j}^{n} V\right)^{2},
$$

one has

$$
\sqrt{k_{n}}\left(\left(\begin{array}{c}
F_{i}^{n} \\
G_{i}^{n} \\
H_{i}^{n}
\end{array}\right)-\left(\begin{array}{c}
\rho \\
1 \\
1
\end{array}\right)\right) \stackrel{w}{\longrightarrow} \mathcal{N}(0, \Sigma) \quad \text { with } \quad \Sigma=\left(\begin{array}{ccc}
1+\rho^{2} & 2 \rho & 2 \rho \\
2 \rho & 2 & 2 \rho^{2} \\
2 \rho & 2 \rho^{2} & 2
\end{array}\right),
$$

and the delta method gives

$$
\sqrt{k_{n}}\left(U_{i}^{n}-\rho\right) \stackrel{w}{\longrightarrow} \mathcal{N}\left(0, v^{2}\right) .
$$

(5.55) can now be derived easily.

Proof of Lemma 3.2 We use Itô's formula again. For any $1 \leq j \leq m_{n}$ we have

$$
\left(X_{\frac{j+k}{n}}-X_{\frac{j+k-1}{n}}\right)^{2}=2 \int_{\frac{j+k-1}{n}}^{\frac{j+k}{n}}\left(\left(X_{s}-X_{\frac{j+k-1}{n}}\right) a_{s}+\sigma_{s}^{2}\right) d s+2 \int_{\frac{j+k-1}{n}}^{\frac{j+k}{n}}\left(X_{s}-X_{\frac{j+k-1}{n}}\right) \sigma_{s} d W_{s},
$$

and thus

$$
\begin{aligned}
& \hat{\sigma}_{\frac{k}{n}}^{2}-\sigma_{\frac{k}{n}}^{2}=\frac{n}{m_{n}} \sum_{j=1}^{m_{n}}\left(\left(X_{\frac{j+k}{n}}-X_{\frac{j+k-1}{n}}\right)^{2}-\frac{1}{n} \sigma_{\frac{k}{n}}^{2}\right) \\
= & \frac{n}{m_{n}} \sum_{j=1}^{m_{n}}\left(\int_{\frac{j+k-1}{n}}^{\frac{j+k}{n}} 2\left(X_{s}-X_{\frac{j+k-1}{n}}\right) a_{s}+\left(\sigma_{s}^{2}-\sigma_{\frac{k}{n}}^{2}\right) d s+2 \int_{\frac{j+k-1}{n}}^{\frac{j+k}{n}}\left(X_{s}-X_{\frac{j+k-1}{n}}\right) \sigma_{s} d W_{s}\right) .
\end{aligned}
$$

From Burkholder's inequality it is obvious that

$$
\frac{n}{m_{n}} \sum_{j=1}^{m_{n}} \int_{\frac{j+k-1}{n}}^{\frac{j+k}{n}} 2\left(X_{s}-X_{\frac{j+k-1}{n}}\right) a_{s} d s=O_{p}\left(n^{-\frac{1}{2}}\right)
$$

and the same way we have

$$
\frac{n}{m_{n}} \sum_{j=1}^{m_{n}} \int_{\frac{j+k-1}{n}}^{\frac{j+k}{n}}\left(\sigma_{s}^{2}-\sigma_{\frac{k}{n}}^{2}\right) d s=O_{p}\left(\sqrt{\frac{m_{n}}{n}}\right)
$$


for the second term. By successive conditioning we obtain finally

$E\left[\left(\frac{n}{m_{n}} \sum_{j=1}^{m_{n}} \int_{\frac{j+k-1}{n}}^{\frac{j+k}{n}}\left(X_{s}-X_{\frac{j+k-1}{n}}\right) \sigma_{s} d W_{s}\right)^{2}\right]=\frac{n^{2}}{m_{n}^{2}} \sum_{j=1}^{m_{n}} \int_{\frac{j+k-1}{n}}^{\frac{j+k}{n}} E\left[\left(X_{s}-X_{\frac{j+k-1}{n}}\right)^{2} \sigma_{s}^{2}\right] d s \leq C \frac{1}{m_{n}}$,

which finishes the proof.

Proof of Theorem 3.4 We will only give a proof of the central limit theorem, as the consistency result follows essentially from the same lines. To this end we start with some additional notation, which in parts is similar to the one used in the proof of Theorem 2.1, but should not be mixed up with it. First, we set $r_{i j}=3 i k_{n}+3 j-1$ and define

$$
\begin{aligned}
\hat{\mu}_{i}^{n} & =\frac{n}{k_{n} l_{n}} \sum_{j=1}^{k_{n}}\left(X_{\frac{r_{i j} l_{n}}{n}}-X_{\frac{\left(r_{i j}-1\right) l_{n}}{n}}\right)^{2} \frac{n}{k_{n} l_{n}} \sum_{j=1}^{k_{n}}\left(\hat{\sigma}_{\frac{r_{i j} l_{n}}{n}}^{2}-\hat{\bar{\sigma}}_{\frac{\left(r_{i j}-1\right) l_{n}}{n}}^{2}\right)^{2}, \\
\mu_{i}^{n} & =\frac{n}{k_{n} l_{n}} \sum_{j=1}^{k_{n}}\left(X_{\frac{r_{i j} l_{n}}{n}}-X_{\frac{\left(r_{i j}-1\right) l_{n}}{n}}\right)^{2} \frac{n}{k_{n} l_{n}} \sum_{j=1}^{k_{n}}\left(\sigma_{\frac{r_{i j} l_{n}}{n}}^{2}-\sigma_{\frac{\left(r_{i j}-1\right) l_{n}}{n}}^{2}\right)^{2} \text {, and } \\
\chi_{i}^{n} & =\frac{n}{k_{n} l_{n}} \sum_{j=1}^{k_{n}} \sigma_{\frac{3 i k_{n} l_{n} l_{n}}{n}}^{2}\left(W_{\frac{r_{i j} l_{n}}{n}}-W_{\frac{\left(r_{i j}-1\right) l_{n}}{n}}\right)^{2} \frac{n}{k_{n} l_{n}} \sum_{j=1}^{k_{n}} \tau_{\frac{3 i k_{n} l_{n}}{n}}^{2}\left(V_{\frac{r_{i j} l_{n}}{n}}-V_{\frac{\left(r_{i j}-1\right) l_{n}}{n}}\right)^{2}
\end{aligned}
$$

for $i=0, \ldots,\left\lfloor\frac{n}{3 k_{n} l_{n}}\right\rfloor-1$, which are the (squared) denominator of $\hat{Z}_{i}^{n}$ and two natural approximations for it. We define similar quantities for its nominator:

$$
\begin{aligned}
\hat{\delta}_{i}^{n} & =\frac{n}{k_{n} l_{n}} \sum_{j=1}^{k_{n}}\left(X_{\frac{r_{i j} l_{n}}{n}}-X_{\frac{\left(r_{i j}-1\right) l_{n}}{n}}\right)\left(\hat{\sigma}_{\frac{r_{i j} l_{n}}{n}}^{2}-\hat{\bar{\sigma}}_{\frac{\left(r_{i j}-1\right) l_{n}}{n}}^{2}\right) \text { and } \\
\delta_{i}^{n} & =\frac{n}{k_{n} l_{n}} \sum_{j=1}^{k_{n}}\left(X_{\frac{r_{i j} l_{n}}{n}}-X_{\frac{\left(r_{i j}-1\right) l_{n}}{n}}\right)\left(\sigma_{\frac{r_{i j} l_{n}}{n}}^{2}-\sigma_{\frac{\left(r_{i j}-1\right) l_{n}}{n}}^{2}\right) .
\end{aligned}
$$

Finally

$$
Z_{n}^{i}=\frac{\frac{1}{k_{n}} \sum_{j=1}^{k_{n}} \frac{n}{l_{n}}\left(X_{\frac{r_{i j} l_{n}}{n}}-X_{\frac{\left(r_{i j}-1\right) l_{n}}{n}}\right)\left(\sigma_{\frac{r_{i j} l_{n}}{n}}^{2}-\sigma_{\frac{\left(r_{i j}-1\right) l_{n}}{n}}^{2}\right)}{\sqrt{\frac{n}{k_{n} l_{n}} \sum_{j=1}^{k_{n}}\left(X_{\frac{r_{i j} l_{n}}{n}}-X_{\frac{\left(r_{i j}-1\right) l_{n}}{n}}\right)^{2} \frac{n}{k_{n} l_{n}} \sum_{j=1}^{k_{n}}\left(\sigma_{\frac{r_{i j} l_{n}}{n}}^{2}-\sigma_{\frac{\left(r_{i j}-1\right) l_{n}}{n}}^{2}\right)^{2}}},
$$

and from Theorem 2.1 we have

$$
\sqrt{\frac{n}{3 l_{n}}} \frac{3 k_{n} l_{n}}{n} \sum_{i=0}^{\left\lfloor\frac{n}{3 k_{n} l_{n}}\right\rfloor-1}\left(Z_{i}^{n}+\frac{Z_{i}^{n}\left(1-\left(Z_{i}^{n}\right)^{2}\right)}{2\left(k_{n}-3\right)}\right) \stackrel{w}{\longrightarrow} \mathcal{N}\left(0, v^{2}\right) .
$$

Our task therefore is to show that the error due to replacing $Z_{i}^{n}$ by $\hat{Z}_{i}^{n}$ is negligible. Since

$$
\hat{Z}_{i}^{n}-Z_{i}^{n}=\frac{\hat{\delta}_{i}^{n}}{\sqrt{\hat{\mu}_{i}^{n}}}-\frac{\delta_{i}^{n}}{\sqrt{\mu_{i}^{n}}}=\frac{\hat{\delta}_{i}^{n}-\delta_{i}^{n}}{\sqrt{\mu_{i}^{n}}}+\frac{\hat{\delta}_{i}^{n}\left(\mu_{i}^{n}-\hat{\mu}_{i}^{n}\right)}{\sqrt{\mu_{i}^{n}} \sqrt{\hat{\mu}_{i}^{n}}\left(\sqrt{\mu_{i}^{n}}+\sqrt{\hat{\mu}_{i}^{n}}\right)},
$$


we prove in a first step that

$$
\sqrt{\frac{n}{l_{n}}} \frac{k_{n} l_{n}}{n} \sum_{i=0}^{\left\lfloor\frac{n}{3 k_{n} l_{n}}\right\rfloor-1} \frac{\hat{\delta}_{i}^{n}-\delta_{i}^{n}}{\sqrt{\mu_{i}^{n}}}=o_{p}(1)
$$

From Burkholder's inequality we have

$$
X_{\frac{r_{i j} l_{n}}{n}}-X_{\frac{\left(r_{i j}-1\right) l_{n}}{n}}=\sigma_{\frac{3 i l_{n} k_{n}}{n}}\left(W_{\frac{r_{i j} l_{n}}{n}}-W_{\frac{\left(r_{i j}-1\right) l_{n}}{n}}\right)+R_{i j}^{n} \quad \text { with } \quad E\left[\left|R_{i j}^{n}\right|^{q}\right] \leq C_{q} k_{n}^{q / 2}\left(\frac{l_{n}}{n}\right)^{q},
$$

and using (5.56) we are able to show

$$
\hat{\sigma}_{\frac{r_{i j} l_{n}}{n}}^{2}-\sigma_{\frac{r_{i j} l_{n}}{n}}^{2}=\psi_{i j}^{n}+S_{i j}^{n} \quad \text { with } \quad E\left[\left|S_{i j}^{n}\right|^{q}\right] \leq C_{q} \frac{\left(l_{n} k_{n} m_{n}\right)^{q / 2}}{n^{q}},
$$

where

$$
\psi_{i j}^{n}=\frac{n}{m_{n}} \sum_{k=r_{i j} l_{n}+1}^{r_{i j} l_{n}+m_{n}}\left(\tau_{\frac{3 i l_{n} k_{n}}{n}} \int_{\frac{k-1}{n}}^{\frac{k}{n}} \int_{\frac{r_{i j} l_{n}}{n}}^{s} d V_{u} d s+2 \sigma_{\frac{3 i l_{n} k_{n}}{n}}^{2} \int_{\frac{k-1}{n}}^{\frac{k}{n}} \int_{\frac{k-1}{n}}^{s} d W_{u} d W_{s}\right) .
$$

A similar decomposition holds for $\frac{\hat{\sigma}_{\left(r_{i j}-1\right) l_{n}}^{2}}{n}-\sigma_{\frac{\left(r_{i j}-1\right) l_{n}}{n}}^{2}$, in which case the approximation is

$$
\phi_{i j}^{n}=\frac{n}{m_{n}} \sum_{k=\left(r_{i j}-1\right) l_{n}-m_{n}}^{\left(r_{i j}-1\right) l_{n}-1}\left(\tau_{\frac{3 i l_{n} k_{n}}{n}} \int_{\frac{k}{n}}^{\frac{k+1}{n}} \int_{\frac{\left(r_{i j}-1\right) l_{n}}{n}}^{s} d V_{u} d s+2 \sigma_{\frac{3 i l_{n} k_{n}}{n}}^{2} \int_{\frac{k}{n}}^{\frac{k+1}{n}} \int_{\frac{k}{n}}^{s} d W_{u} d W_{s}\right) .
$$

Thus with

$$
D_{i}^{n}=\frac{n}{k_{n} l_{n}} \sum_{j=1}^{k_{n}} \psi_{i j}^{n} \sigma_{\frac{3 i l_{n} k_{n}}{n}} \int_{\frac{\left(r_{i j}-1\right) l_{n}}{n}}^{\frac{r_{i j} l_{n}}{n}} d W_{s} \quad \text { and } \quad E_{i}^{n}=\frac{n}{k_{n} l_{n}} \sum_{j=1}^{k_{n}} \phi_{i j}^{n} \sigma_{\frac{3 i l_{n} k_{n}}{n}} \int_{\frac{\left(r_{i j}-1\right) l_{n}}{n}}^{\frac{r_{i j} l_{n}}{n}} d W_{s}
$$

we can proceed as in (5.22)-(5.25): We have to show

$$
\begin{aligned}
& P\left(\min _{i} \sqrt{\mu_{i}^{n}}<K-\delta\right) \rightarrow 0 \text { for any } 0<\delta<K, \\
& k_{n} \sqrt{\frac{l_{n}}{n}} \sum_{i=0}^{\left\lfloor\frac{n}{3 l n k_{n}}\right\rfloor-1} E\left[\left|\hat{\delta}_{i}^{n}-\delta_{i}^{n}-\left(D_{i}^{n}-E_{i}^{n}\right)\right|\right] \rightarrow 0, \\
& k_{n} \sqrt{\frac{l_{n}}{n}} \sum_{i=0}^{\left\lfloor\frac{n}{3 l n}\right\rfloor-1} \frac{D_{i}^{n}-E_{i}^{n}}{\sqrt{\chi_{i}^{n}}} \stackrel{P}{\longrightarrow} 0 \text { and } \\
& k_{n} \sqrt{\frac{l_{n}}{n}} \sum_{i=0}^{\left\lfloor\frac{n}{3 l_{n} k_{n}}\right\rfloor-1}\left(\frac{D_{i}^{n}-E_{i}^{n}}{\sqrt{\mu_{i}^{n}}}-\frac{D_{i}^{n}-E_{i}^{n}}{\sqrt{\chi_{i}^{n}}}\right) \stackrel{P}{\longrightarrow} 0 .
\end{aligned}
$$

(5.65) follows from (5.22), whereas we conclude

$$
k_{n} \sqrt{\frac{l_{n}}{n}} \sum_{i=0}^{\left\lfloor\frac{n}{3 l_{n} k_{n}}\right\rfloor-1} E\left[\left|\hat{\delta}_{i}^{n}-\delta_{i}^{n}-\left(D_{i}^{n}-E_{i}^{n}\right)\right|\right] \leq C \sqrt{\frac{n}{l_{n}}} \frac{n}{l_{n}} \frac{\left(k_{n} m_{n}\right)^{1 / 2} l_{n}}{n^{3 / 2}}=C\left(\frac{k_{n} m_{n}}{l_{n}}\right)^{1 / 2} \rightarrow 0
$$


from (5.62) and (5.63) and by assumption on $k_{n}$, which gives (5.66). (5.67) can be shown in the same way as (5.24), and one uses that the quantities $D_{i}^{n}, E_{i}^{n}$ and $\chi_{i}^{n}$ are defined on disjoint intervals. From the independence of increments of a Brownian motion one obtains (in order to have an existing expected value, $n$ has to be large enough again)

$$
E\left[\frac{D_{i}^{n}-E_{i}^{n}}{\sqrt{\chi_{i}^{n}}} \mid \mathcal{F}_{\frac{3 i l_{n} k_{n}}{n}}\right]=0
$$

and thus $E\left[\left(D_{i}^{n}-E_{i}^{n}\right)^{4}\right]<C\left(\frac{m_{n}}{k_{n} l_{n}}+\frac{n}{k_{n} l_{n} m_{n}}\right)^{2}$ plus Hölder's inequality gives

$$
E\left[\left(k_{n} \sqrt{\frac{l_{n}}{n}} \sum_{i=0}^{\left\lfloor\frac{n}{3 l_{n} k_{n}}\right\rfloor-1} \frac{D_{i}^{n}-E_{i}^{n}}{\sqrt{\chi_{i}^{n}}}\right)^{2}\right]<C\left(\frac{m_{n}}{l_{n}}+\frac{n}{l_{n} m_{n}}\right) \rightarrow 0 .
$$

Finally, we can use $E\left[\left(\chi_{i}^{n}-\mu_{i}^{n}\right)^{2}\right]<C \frac{k_{n} l_{n}}{n}$ from (5.41) in order to obtain

$$
k_{n} \sqrt{\frac{l_{n}}{n}} \sum_{i=0}^{\left\lfloor\frac{n}{3 l_{n} k_{n}}\right\rfloor-1} E\left[\left|\left(D_{i}^{n}-E_{i}^{n}\right)\left(\chi_{i}^{n}-\mu_{i}^{n}\right)\right|\right] \leq C \sqrt{\frac{n}{l_{n}}} \sqrt{\frac{m_{n}}{k_{n} l_{n}}} \sqrt{\frac{k_{n} l_{n}}{n}}=C \sqrt{\frac{m_{n}}{l_{n}}} \rightarrow 0 .
$$

As in the proof of (5.25) this is sufficient to derive (5.68) and thus (5.61).

We come to the analysis of

$$
\frac{\hat{\delta}_{i}^{n}\left(\mu_{i}^{n}-\hat{\mu}_{i}^{n}\right)}{\sqrt{\mu_{i}^{n}} \sqrt{\hat{\mu}_{i}^{n}}\left(\sqrt{\mu_{i}^{n}}+\sqrt{\hat{\mu}_{i}^{n}}\right)},
$$

and we define another three terms, which will be used to approximate the nominator above, namely

$$
\begin{aligned}
\gamma_{i}^{n} & =\frac{n}{k_{n} l_{n}} \sum_{j=1}^{k_{n}} \sigma_{\frac{3 i l_{n} k_{n}}{n}}\left(W_{\frac{r_{i j} l_{n}}{n}}-W_{\frac{\left(r_{i j}-1\right) l_{n}}{n}}\right) \tau_{\frac{3 i l_{n} k_{n}}{n}}\left(V_{\frac{r_{i j} l_{n}}{n}}-V_{\frac{\left(r_{i j}-1\right) l_{n}}{n}}\right) \\
\tilde{\mu}_{i}^{n} & =\frac{n}{k_{n} l_{n}} \sum_{j=1}^{k_{n}} \sigma_{\frac{3 i l_{n} k_{n}}{n}}^{2}\left(W_{\frac{r_{i j} l_{n}}{n}}-W_{\frac{\left(r_{i j}-1\right) l_{n}}{n}}\right)^{2} \frac{n}{k_{n} l_{n}} \sum_{j=1}^{k_{n}}\left(-\left(\psi_{i j}^{n}\right)^{2}-\left(\phi_{i j}^{n}\right)^{2}\right) \quad \text { and } \\
\bar{\mu}_{i}^{n} & =\frac{n^{2}}{k_{n}^{2} l_{n}^{2}} \sum_{j=1}^{k_{n}} \sigma_{\frac{3 i l_{n} k_{n}}{n}}^{2}\left(\int_{\frac{\left(r_{i j}-1\right) l_{n}}{n}}^{\frac{r_{i j} l_{n}}{n}} d W_{s}\right)^{2} \sum_{j=1}^{k_{n}}\left(-\left(\psi_{i j}^{n}\right)^{2}-\left(\phi_{i j}^{n}\right)^{2}-2\left(\psi_{i j}^{n}+\phi_{i j}^{n}\right) \frac{3 i l_{n} k_{n}}{n} \int_{\frac{\left(r_{i j}-1\right) l_{n}}{n}}^{\frac{r_{i j} l_{n}}{n}} d V_{s}\right) .
\end{aligned}
$$

with $\psi_{i j}^{n}$ and $\phi_{i j}^{n}$ as in (5.63) and (5.64), respectively. The second step now consists of establishing

$$
\sqrt{\frac{n}{l_{n}}} \frac{k_{n} l_{n}}{n} \sum_{i=0}^{\left\lfloor\frac{n}{3 k_{n} l_{n}}\right\rfloor-1}\left(\frac{\hat{\delta}_{i}^{n}\left(\mu_{i}^{n}-\hat{\mu}_{i}^{n}\right)}{\sqrt{\mu_{i}^{n}} \sqrt{\hat{\mu}_{i}^{n}}\left(\sqrt{\mu_{i}^{n}}+\sqrt{\hat{\mu}_{i}^{n}}\right)}-\frac{\gamma_{i}^{n} \tilde{\mu}_{i}^{n}}{2\left(\chi_{i}^{n}\right)^{3 / 2}}\right)=o_{p}(1),
$$


whose proof will be split into three parts, namely

$$
\begin{aligned}
& \sqrt{\frac{n}{l_{n}}} \frac{k_{n} l_{n}}{n} \sum_{i=0}^{\left\lfloor\frac{n}{3 k_{n} l_{n}}\right\rfloor-1} \frac{\hat{\delta}_{i}^{n}\left(\mu_{i}^{n}-\hat{\mu}_{i}^{n}\right)-\gamma_{i}^{n} \bar{\mu}_{i}^{n}}{\sqrt{\mu_{i}^{n}} \sqrt{\hat{\mu}_{i}^{n}}\left(\sqrt{\mu_{i}^{n}}+\sqrt{\hat{\mu}_{i}^{n}}\right)}=o_{p}(1), \\
& \sqrt{\frac{n}{l_{n}}} \frac{k_{n} l_{n}}{n} \sum_{i=0}^{\left\lfloor\frac{n}{3 k_{n} l_{n}}\right\rfloor-1}\left(\frac{\gamma_{i}^{n} \bar{\mu}_{i}^{n}}{\sqrt{\mu_{i}^{n}} \sqrt{\hat{\mu}_{i}^{n}}\left(\sqrt{\mu_{i}^{n}}+\sqrt{\hat{\mu}_{i}^{n}}\right)}-\frac{\gamma_{i}^{n} \bar{\mu}_{i}^{n}}{2\left(\chi_{i}^{n}\right)^{3 / 2}}\right)=o_{p}(1), \\
& \sqrt{\frac{n}{l_{n}}} \frac{k_{n} l_{n}}{n} \sum_{i=0}^{\left\lfloor\frac{n}{3 k_{n} l_{n}}\right\rfloor-1} \frac{\gamma_{i}^{n}\left(\bar{\mu}_{i}^{n}-\tilde{\mu}_{i}^{n}\right)}{2\left(\chi_{i}^{n}\right)^{3 / 2}}=o_{p}(1) .
\end{aligned}
$$

Note first that one obtains

$$
E\left[\left|\left(\sigma_{\frac{r_{i j} l_{n}}{n}}^{2}-\sigma_{\frac{\left(r_{i j}-1\right) l_{n}}{n}}^{2}\right)^{2}-\left(\hat{\sigma}_{\frac{r_{i j} l_{n}}{n}}^{2}-\hat{\bar{\sigma}}_{\frac{\left(r_{i j}-1\right) l_{n}}{n}}^{2}\right)^{2}\right|^{q}\right] \leq C_{q} \frac{\left(m_{n} l_{n}\right)^{q / 2}}{n^{q}}
$$

as the binomial theorem gives a natural decomposition of the left hand side of the preceding display into five terms, whose orders are simple to compute. We use this result to prove

$$
P\left(\min _{i} \sqrt{\hat{\mu}_{i}^{n}}<K-\delta\right) \rightarrow 0 \text { for any } 0<\delta<K
$$

first. Recall (5.22) and its proof. It is then easy to see that it suffices to verify

$$
P\left(\min _{i} \frac{n}{k_{n} l_{n}}\left|\sum_{j=1}^{k_{n}}\left(\sigma_{\frac{r_{i j} l_{n}}{n}}^{2}-\sigma_{\frac{\left(r_{i j}-1\right) l_{n}}{n}}^{2}\right)^{2}-\left(\hat{\sigma}_{\frac{r_{i j} l_{n}}{n}}^{2}-\hat{\bar{\sigma}}_{\frac{\left(r_{i j}-1\right) l_{n}}{n}}^{2}\right)^{2}\right|>\frac{\delta}{2}\right) \rightarrow 0,
$$

which thus follows from Markov's inequality, in the same way as the corresponding result in the proof of (5.22). This claim helps in order to obtain (5.71), since similar to the proof of Lemma 5.1 it is now sufficient to establish

$$
\sqrt{\frac{n}{l_{n}}} E\left[\left|\hat{\delta}_{i}^{n}\left(\mu_{i}^{n}-\hat{\mu}_{i}^{n}\right)-\gamma_{i}^{n} \bar{\mu}_{i}^{n}\right|\right] \rightarrow 0 \quad \text { uniformly in } i .
$$

Showing (5.76) is tedious. The main idea is that one exchanges the quantities in $\hat{\delta}_{i}^{n}\left(\mu_{i}^{n}-\hat{\mu}_{i}^{n}\right)$ in several steps by less complex ones, where the error of each replacement is small. Precisely, we use (5.62) and (5.63) plus (5.74) in order to first replace any increment of $X$ by $\frac{\sigma_{i i l_{n} k_{n}}}{n}$ times the corresponding increment of $W$. Then the overall size of the error due to this approximation is of order $\sqrt{\frac{k_{n} m_{n}}{l_{n}}} \rightarrow 0$. The same error arises, if we replace

$$
\left(\hat{\sigma}_{\frac{r_{i j} l_{n}}{n}}^{2}-\hat{\bar{\sigma}}_{\frac{\left(r_{i j}-1\right) l_{n}}{n}}^{2}\right) \quad \text { by } \quad \psi_{i j}^{n}+\tau_{\frac{3 i l_{n} k_{n}}{n}} \int_{\frac{\left(r_{i j}-1\right) l_{n}}{n}}^{\frac{r_{i j} l_{n}}{n}} d V_{s}-\phi_{i j}^{n}
$$

afterwards, and using (5.63) and (5.64) we can finally define similar approximations for each of the five terms in the decomposition of (5.74), giving again an overall error of size $\sqrt{\frac{k_{n} m_{n}}{l_{n}}}$. This new term is already close to $\gamma_{i}^{n} \bar{\mu}_{i}^{n}$, but still includes an additional term involving the product of $\psi_{i j}^{n}$ and 
$\phi_{i j}^{n}$. However, using that both $\psi_{i j}^{n}, \phi_{i j}^{n}$ and the $d W_{s}-, d V_{s}$-integrals are living on disjoint intervals, a simple calculation gives that this additional term is of small order, that is on the whole

$$
\frac{n}{l_{n}} E\left[\left|\hat{\delta}_{i}^{n}\left(\mu_{i}^{n}-\hat{\mu}_{i}^{n}\right)-\gamma_{i}^{n} \tilde{\mu}_{i}^{n}\right|^{2}\right] \leq C\left(\frac{k_{n} m_{n}}{l_{n}}+\frac{n m_{n}^{2}}{l_{n}^{3} k_{n}}\right) \leq C\left(\frac{k_{n} m_{n}}{l_{n}}+\frac{1}{k_{n}}\right) \rightarrow 0,
$$

and thus (5.71) follows. For the proof of (5.72) note that

$$
\frac{\gamma_{i}^{n} \bar{\mu}_{i}^{n}}{\sqrt{\mu_{i}^{n}} \sqrt{\hat{\mu}_{i}^{n}}\left(\sqrt{\mu_{i}^{n}}+\sqrt{\hat{\mu}_{i}^{n}}\right)}-\frac{\gamma_{i}^{n} \bar{\mu}_{i}^{n}}{2\left(\chi_{i}^{n}\right)^{3 / 2}}
$$

can be decomposed in a similar way as the difference in (5.50), which forces us to show

$$
\sqrt{\frac{n}{l_{n}}} E\left[\left|\gamma_{i}^{n} \bar{\mu}_{i}^{n}\left(\chi_{i}^{n}-\mu_{i}^{n}\right)\left(\sqrt{\mu_{i}^{n}}+\sqrt{\hat{\mu}_{i}^{n}}\right) \sqrt{\hat{\mu}_{i}^{n}}\right|\right] \rightarrow 0 \text { uniformly in } i
$$

and several related results, where the main difference is that $\left(\chi_{i}^{n}-\mu_{i}^{n}\right)$ must sometimes be replaced by $\left(\chi_{i}^{n}-\hat{\mu}_{i}^{n}\right)$. However, we use (5.41) and derive

$$
E\left[\left|\mu_{i}^{n}-\hat{\mu}_{i}^{n}\right|^{q}\right] \leq C_{q}\left(\frac{m_{n}}{l_{n}}\right)^{q / 2}, \quad E\left[\left|\gamma_{i}^{n}\right|^{q}\right] \leq C_{q}, \quad E\left[\left|\bar{\mu}_{i}^{n}\right|^{q}\right] \leq C_{q}\left(\frac{m_{n}}{k_{n} l_{n}}\right)^{q / 2}
$$

from (5.74) and some careful computations, thus (5.72) follows from Hölder's inequality. Finally,

$$
\begin{aligned}
\gamma_{i}^{n}\left(\bar{\mu}_{i}^{n}-\tilde{\mu}_{i}^{n}\right)=\frac{n^{3}}{k_{n}^{3} l_{n}^{3}} \sum_{j=1}^{k_{n}} \sigma_{\frac{3 i l_{n} k_{n}}{n}}^{3} \frac{\tau_{\frac{3 i l_{n} k_{n}}{n}}^{2} \int_{\frac{\left(r_{i j}-1\right) l_{n}}{n}}^{\frac{r_{i j} l_{n}}{n}} d W_{s} \int_{\frac{\left(r_{i j}-1\right) l_{n}}{n}}^{\frac{r_{i j} l_{n}}{n}} d V_{s} \sum_{k=1}^{k_{n}}\left(\int_{\frac{\left(r_{i k}-1\right) l_{n}}{n}}^{\frac{r_{i k} l_{n}}{n}} d W_{s}\right)^{2}}{} \\
\sum_{l=1}^{k_{n}}\left(-2\left(\psi_{i l}^{n}+\phi_{i l}^{n}\right) \int_{\frac{\left(r_{i l}-1\right) l_{n}}{n}}^{\frac{r_{i l} l_{n}}{n}} d V_{s}\right)=O_{p}\left(\frac{m_{n}}{l_{n} k_{n}}\right) .
\end{aligned}
$$

Introducing $\mathcal{G}_{i}^{n}$, which is the smallest $\sigma$-algebra containing $\mathcal{F}_{\frac{3 i l_{n} k_{n}}{n}}$ and such that the increments of $W$ and $V$ on $\left[\frac{\left(r_{i j}-1\right) l_{n}}{n}, \frac{r_{i j} l_{n}}{n}\right], j=1, \ldots, k_{n}$, are $\mathcal{G}_{i}^{n}$-measurable we obtain

$$
E\left[\frac{\gamma_{i}^{n}\left(\bar{\mu}_{i}^{n}-\tilde{\mu}_{i}^{n}\right)}{2\left(\chi_{i}^{n}\right)^{3 / 2}} \mid \mathcal{F}_{\frac{3 i l_{n} k_{n}}{n}}\right]=E\left[E\left(\frac{\gamma_{i}^{n}\left(\bar{\mu}_{i}^{n}-\tilde{\mu}_{i}^{n}\right)}{2\left(\chi_{i}^{n}\right)^{3 / 2}} \mid \mathcal{G}_{i}^{n}\right) \mid \mathcal{F}_{\frac{3 i l_{n} k_{n}}{n}}\right]=0
$$

since $\psi_{i l}^{n}$ and $\phi_{i l}^{n}$ are centered and independent of $\mathcal{G}_{i}^{n}$ for each $l$. Therefore

$$
E\left[\left(\sqrt{\frac{n}{l_{n}}} \frac{k_{n} l_{n}}{n} \sum_{i=0}^{\left\lfloor\frac{n}{3 k_{n} l_{n}}\right\rfloor-1} \frac{\gamma_{i}^{n}\left(\bar{\mu}_{i}^{n}-\tilde{\mu}_{i}^{n}\right)}{2\left(\chi_{i}^{n}\right)^{3 / 2}}\right)^{2}\right] \leq C \frac{k_{n}^{2} l_{n}}{n} \sum_{i=0}^{\left\lfloor\frac{n}{3 k_{n} l_{n}}\right\rfloor-1} E\left[\left(\frac{\gamma_{i}^{n}\left(\bar{\mu}_{i}^{n}-\tilde{\mu}_{i}^{n}\right)}{2\left(\chi_{i}^{n}\right)^{3 / 2}}\right)^{2}\right] \leq C \frac{m_{n}}{l_{n}} \rightarrow 0
$$

follows from the Cauchy-Schwarz inequality. This proves (5.73), thus (5.70).

In a third step we establish

$$
\sqrt{\frac{n}{l_{n}}} \frac{l_{n}}{n} \sum_{i=0}^{\left\lfloor\frac{n}{3 k_{n} l_{n}}\right\rfloor-1}\left(\left(\hat{Z}_{i}^{n}\right)^{3}-\left(Z_{i}^{n}\right)^{3}\right) \stackrel{P}{\longrightarrow} 0
$$


which can be shown in a similar way as the corresponding result in (2.11). Precisely, from the same expansion as in (5.52) plus the decomposition (5.60) and the Cauchy-Schwarz inequality it remains to prove

$$
\sqrt{\frac{l_{n}}{n}} \sum_{i=0}^{\left\lfloor\frac{n}{3 k_{n} l_{n}}\right\rfloor-1}\left(E\left[\left|\hat{\delta}_{i}^{n}-\delta_{i}^{n}\right|^{2}\right]^{1 / 2}+E\left[\left|\hat{\mu}_{i}^{n}-\mu_{i}^{n}\right|^{2}\right]^{1 / 2}\right) \rightarrow 0 .
$$

However, we have

$$
E\left[\left|\hat{\delta}_{i}^{n}-\delta_{i}^{n}\right|^{2}\right]^{1 / 2}+E\left[\left|\hat{\mu}_{i}^{n}-\mu_{i}^{n}\right|^{2}\right]^{1 / 2} \leq C \sqrt{\frac{m_{n}}{l_{n}}}
$$

using (5.78) and Lemma 3.2, and since

$$
\frac{\sqrt{n m_{n}}}{l_{n} k_{n}} \leq C \frac{n^{\frac{1}{12}}}{k_{n}} \rightarrow 0
$$

from (3.16), (5.80) and (5.79) follow.

Let us put things together. (5.61), (5.70), (5.79) and (5.59) show that the final part in the proof of Theorem 3.4 consists of establishing

$$
E\left[\left(\frac{k_{n} l_{n}}{n} \sum_{i=0}^{\left\lfloor\frac{n}{k_{n} l_{n}}\right\rfloor-1} \sqrt{\frac{n}{l_{n}}} \frac{\gamma_{i}^{n} \tilde{\mu}_{i}^{n}}{2\left(\chi_{i}^{n}\right)^{3 / 2}}\right)^{2}\right]<C \frac{1}{k_{n}} \rightarrow 0 .
$$

Set $s_{i}^{n}=\frac{\sigma_{3 i l_{n} k_{n}}}{n} \frac{3 i l_{n} k_{n}}{n}$. As the correlation $\rho$ is zero, it is easy to see that both

$$
E\left[\left(\sqrt{\frac{n}{l_{n}}} \gamma_{i}^{n} \tilde{\mu}_{i}^{n}\right)^{4} \mid \mathcal{F}_{\frac{3 i l_{n} k_{n}}{n}}\right]=O_{p}\left(\frac{1}{k_{n}^{2}}\right) \quad \text { and } \quad E\left[\left(\chi_{i}^{n}-s_{i}^{n}\right)^{4} \mid \mathcal{F}_{\frac{3 i l_{n} k_{n}}{n}}\right]=O_{p}\left(\frac{1}{k_{n}^{2}}\right)
$$

hold. Also, for a twice continuously differentiable function $f$ on $\mathbb{R}^{2}$ a Taylor expansion gives

$$
f(x, y)=f\left(0, s_{i}^{n}\right)+\left(x, y-s_{i}^{n}\right) \nabla f\left(\xi_{i}^{n}, \vartheta_{i}^{n}\right)
$$

with $\left|\xi_{i}^{n}\right| \leq|x|$ and $\left|\vartheta_{i}^{n}-s_{i}^{n}\right| \leq\left|y-s_{i}^{n}\right|$. Setting $f(x, y)=\frac{x}{2 y^{3 / 2}}$ and inserting $\sqrt{\frac{n}{l_{n}}} \gamma_{i}^{n} \tilde{\mu}_{i}^{n}$ and $\chi_{i}^{n}$ for $x$ and $y$ we obtain from (5.82) and the Cauchy-Schwarz inequality

$$
\left|E\left[\sqrt{\frac{n}{l_{n}}} \frac{\gamma_{i}^{n} \tilde{\mu}_{i}^{n}}{2\left(\chi_{i}^{n}\right)^{3 / 2}} \mid \mathcal{F}_{\frac{3 i l_{n} k_{n}}{n}}\right]\right|=O_{p}\left(\frac{1}{\sqrt{k_{n}}}\right) .
$$

The proof of (5.81) is simple now.

\section{References}

[1] Aït-Sahalia, Y., R. Kimmel. (2007): Maximum likelihood estimation of stochastic volatility models. Journal of Financial Economics 134, 507-551. 
[2] Barndorff-Nielsen, O. E., S. E. Graversen, J. Jacod, M. Podolskij, N. Shephard. (2006): A central limit theorem for realised power and bipower variations of continuous semimartingales. In: Kabanov, Yu., R. Liptser, J. Stoyanov. (Eds.), From Stochastic Calculus to Mathematical Finance. Festschrift in Honour of A. N. Shiryaev. Springer-Verlag, Berlin, 33-68.

[3] Barndorff-Nielsen, O. E., P. R. Hansen, A. Lunde, N. Shephard. (2008). Designing realised kernels to measure the ex-post variation of equity prices in the presence of noise. Econometrica $76,1481-1536$.

[4] Barndorff-Nielsen, O. E., N. Shephard. (2004): Econometric analysis of realized covariation: High frequency based covariance, regression, and correlation in financial economics. Econometrica, 72, 885-925.

[5] Barndorff-Nielsen, O., N. Shephard, M. Winkel. (2006): Limit theorems for multipower variation in the presence of jumps. Stoch. Proc. Appl., 116, 796-806.

[6] Hayashi, T., N. Yoshida. (2005): On covariance estimation of non-synchronously observed diffusion processes. Bernoulli, 11, 359-379.

[7] Heston, S. J. (1993): A closed-form solution for options with stochastic volatility with applications to bonds and currency options. Review of Financial Studies, 6 327-343.

[8] Hull, J., A. White. (1987): The pricing of options on assets with stochastic volatilities. J. Finance 3, 281-300.

[9] Jacod, J. (2008): Asymptotic properties of realized power variations and related functionals of semimartingales. Stoch. Proc. Appl., 118, 517-559.

[10] Jacod, J., M. Podolskij, M. Vetter. (2010): Limit theorems for moving averages of discretized processes plus noise. Ann. Statist. 38, 1478-1545.

[11] Jacod, J., A. Shiryaev. (2003): Limit theorems for stochastic processes. Springer-Verlag, Berlin.

[12] Mancini, C. (2009): Non-parametric estimation for models with stochastic diffusion coefficients and jumps. Scand. J. Statist., 36, 270-296.

[13] Olkin, I., J. Pratt. (1958): Unbiased estimation of certain correlation coefficients. Ann. Math. Statist., 29, 201-211.

[14] Schöbel, R., J. Zhu. (1999): Stochastic volatility with an Ornstein-Uhlenbeck process: An extension. Europ. Finance Review 3, 23-46.

[15] Stein, E., J. Stein. (1991): Stock price distributions with stochastic volatility: An analytical approach. Rev. Financial Studies 4, 727-752.

[16] Zhang, L., P. A. Mykland, Y. Aït-Sahalia. (2005). A tale of two time scales: Determining integrated volatility with noisy high-frequency data. J. Amer. Statist. Assoc. 472, 1394-1411. 


\begin{tabular}{|c||c|c|c||c|c|c||c|c|c|}
\hline \multicolumn{1}{|c||}{$\rho$} & \multicolumn{3}{c||}{-0.5} & \multicolumn{3}{c||}{0} & \multicolumn{3}{c|}{0.5} \\
\hline$n / \alpha$ & .025 & .05 & .1 & .025 & .05 & .1 & .025 & .05 & .1 \\
\hline 125 & .0632 & .0985 & .1599 & .0465 & .0789 & .1402 & .0533 & .0896 & .1471 \\
1000 & .0267 & .0508 & .0974 & .0293 & .059 & .1127 & .0457 & .084 & .1487 \\
3375 & .025 & .048 & .0927 & .0312 & .0586 & .1106 & .0372 & .0718 & .1342 \\
8000 & .0237 & .0434 & .0881 & .0272 & .0573 & .1067 & .0389 & .0705 & .1321 \\
15625 & .0239 & .0495 & .0926 & .0307 & .0597 & .1099 & .0376 & .0725 & .1316 \\
27000 & .0208 & .0404 & .0843 & .0304 & .0541 & .1072 & .0358 & .0693 & .1283 \\
\hline
\end{tabular}

Table 1: Simulated quantiles of the statistic from Corollary 2.3 for various choices of the sample size $n$ and the correlation parameter $\rho$.

\begin{tabular}{|c||c|c||c|c||c|c||c|c|}
\hline \multicolumn{1}{|c||}{$\rho$} & \multicolumn{2}{c||}{-0.2} & \multicolumn{2}{c||}{-0.1} & \multicolumn{2}{c||}{0.1} & \multicolumn{2}{c|}{0.2} \\
\hline$n / \alpha$ & .05 & .1 & .05 & .1 & .05 & .1 & .05 & .1 \\
\hline 125 & .6046 & .7041 & .2422 & .3389 & .2343 & .3298 & .6097 & .7088 \\
1000 & 1 & 1 & .8764 & .9262 & .8691 & .9218 & .9999 & 1 \\
\hline
\end{tabular}

Table 2: Rejection probabilities of the test from Corollary 2.3 under the null hypothesis $\rho=0$ and for various choices of the sample size $n$ and the alternative $\rho$.

\begin{tabular}{|c|c|c||c|c||c|c||c|c|}
\hline \multicolumn{3}{|c||}{$\rho$} & \multicolumn{2}{c||}{-0.5} & \multicolumn{2}{c||}{0} & \multicolumn{2}{c|}{0.5} \\
\hline$n$ & $m_{n}$ & $k_{n}$ & bias & std. dev. & bias & std. dev. & bias & std. dev. \\
\hline 8000 & 66 & 5 & -.0148 & .1462 & -.0017 & .1802 & .0152 & .1453 \\
15625 & 85 & 6 & -.0081 & .1143 & .002 & .1435 & .01 & .1128 \\
27000 & 115 & 7 & -.0055 & .0953 & .0005 & .1223 & .0058 & .0962 \\
\hline
\end{tabular}

Table 3: Bias and standard deviation of $\hat{\rho}-\rho$ for various choices of the sample size $n$ (and $m_{n}$ and $\left.k_{n}\right)$ and the correlation parameter $\rho$.

\begin{tabular}{|c|c|c|c||c|c|c||}
\hline$n$ & $l_{n}$ & $m_{n}$ & $k_{n}$ & .025 & .05 & .1 \\
\hline 8000 & 140 & 53 & 4 & .0716 & .1067 & .1631 \\
15625 & 190 & 66 & 5 & .05 & .0814 & .1395 \\
27000 & 240 & 82 & 6 & .0415 & .0712 & .1269 \\
\hline
\end{tabular}

Table 4: Simulated quantiles of the $z$-transformed version of (3.18) for $\rho=0$ and various choices of the sample size $n$ (and $l_{n}, m_{n}$ and $k_{n}$ ). 


